\title{
Galactic secondary positron flux at the Earth
}

\author{
T. Delahaye ${ }^{1,2}$, R. Lineros ${ }^{2}$, F. Donato ${ }^{2}$, N. Fornengo ${ }^{2}$, J. Lavalle $^{2}$, P. Salati ${ }^{1}$, and R. Taillet ${ }^{1}$ \\ 1 LAPTH, Université de Savoie, CNRS, BP 110, 74941 Annecy-le-Vieux Cedex, France \\ 2 Dipartimento di Fisica Teorica, Università di Torino \& INFN - Sezione di Torino, via P. Giuria 1, 10122 Torino, Italy \\ e-mail: lineros@to.infn.it \\ Received 10 October 2008 / Accepted 4 March 2009
}

\section{ABSTRACT}

\begin{abstract}
Context. Secondary positrons are produced by spallation of cosmic rays within the interstellar gas. Measurements have been typically expressed in terms of the positron fraction, which exhibits an increase above $10 \mathrm{GeV}$. Many scenarios have been proposed to explain this feature, among them some additional primary positrons originating from dark matter annihilation in the Galaxy.

Aims. The PAMELA satellite has provided high quality data that has enabled high accuracy statistical analyses to be made, showing that the increase in the positron fraction extends up to about $100 \mathrm{GeV}$. It is therefore of paramount importance to constrain theoretically the expected secondary positron flux to interpret the observations in an accurate way.

Methods. We focus on calculating the secondary positron flux by using and comparing different up-to-date nuclear cross-sections and by considering an independent model of cosmic ray propagation. We carefully study the origins of the theoretical uncertainties in the positron flux.

Results. We find the secondary positron flux to be reproduced well by the available observations, and to have theoretical uncertainties that we quantify to be as large as about one order of magnitude. We also discuss the positron fraction issue and find that our predictions may be consistent with the data taken before PAMELA. For PAMELA data, we find that an excess is probably present after considering uncertainties in the positron flux, although its amplitude depends strongly on the assumptions made in relation to the electron flux. By fitting the current electron data, we show that when considering a soft electron spectrum, the amplitude of the excess might be far lower than usually claimed.

Conclusions. We provide fresh insights that may help to explain the positron data with or without new physical model ingredients. PAMELA observations and the forthcoming AMS-02 mission will allow stronger constraints to be aplaced on the cosmic-ray transport parameters, and are likely to reduce drastically the theoretical uncertainties.
\end{abstract}

Key words. ISM: cosmic rays

\section{Introduction}

Among the different particles observed in cosmic rays, positrons still raise unanswered questions. Cosmic positrons are created by spallation reactions of cosmic ray nuclei with interstellar matter and propagate in a diffusive mode, because of their interaction with the turbulent component of the Galactic magnetic field. The expected flux of positrons can be calculated from the observed cosmic ray nuclei fluxes, using the relevant nuclear physics and solving the diffusion equation.

The HEAT experiment (Barwick et al. 1997; Beatty et al. 2004) showed that the positron fraction (the ratio of the positron to the total electron-positron fluxes) possibly exhibits an unexpected bump in the $10 \mathrm{GeV}$ region of the spectrum. Although this bump could be due to some unknown systematic effect, the HEAT result has triggered many explanations. For instance, Moskalenko \& Strong (1998) suggested that an interstellar nucleon spectrum harder than that expected could explain the excess. Many works also focused on the dark matter hypothesis, the bump being due to a primary contribution from the annihilation of dark matter particles. The positron excess expected in this framework is very uncertain, because the nature of dark matter is unknown, and the propagation of positrons involves physical quantities that currently are also not precisely known. The related astrophysical uncertainties were calculated and quantified in Delahaye et al. (2008), where it was shown that they may be significant, especially in the low energy part of the spectrum, a property common also to the antiproton (e.g. Donato et al. 2004) and the antideuteron (Donato et al. 2000, 2008) signals. For positrons, sizeable fluxes from dark matter annihilation are typically possible if dark matter overdensities are locally present, which is usually coded into the socalled "boost factor". A detailed analysis of the admissible boost factors for positrons and antiprotons was performed by Lavalle et al. (2008b), who showed that boost factors are typically confined to less than about a factor of 10-20. Computing the antimatter fluxes directly in the frame of a cosmological $N$-body simulation leads to the same conclusions (Lavalle et al. 2008a).

The PAMELA experiment (Picozza et al. 2007) has released its first results on the positron fraction for energies ranging from $1.5 \mathrm{GeV}$ to $100 \mathrm{GeV}$ and with a large statistics (Adriani et al. 2008). The positron fraction is observed to rise steadily for energies above $10 \mathrm{GeV}$, reinforcing the possibility that an excess is actually present. It is therefore timely and crucial to complete a novel analysis of the positron flux, including a robust estimation of the accuracy of the theoretical determination. The calculation of the uncertainties affecting the standard spallationinduced positron population is especially important when unexpected distortions are observed in experimental data, to address the issue in a more robust way. This and an analysis of the positron signal from dark matter annihilation and its astrophysical uncertainties (Delahaye et al. 2008), will set the proper basis for discussing in detail the experimental results.

The uncertainties in the positron flux have several origins. First, the cosmic ray nuclei measurements have their 
experimental uncertainties, which then affect the predictions of induced secondary fluxes, such as positrons. Second, various modelings of the nuclear cross-sections involved in the positron production mechanism are available, and they are not in complete agreement with each other, implying a range of theoretical variation. Third, the uncertainties in the propagation parameters involved in the diffusion equation were thoroughly studied (Maurin et al. 2001). A detailed analysis of their impact on the secondary positron flux is therefore needed.

In this paper, we therefore study the secondary positron production and transport in the Galaxy, with emphasis on determining the various sources of uncertainties, namely: (i) the nuclear cross-sections; (ii) the effect induced by the primary injection spectra; (iii) the local interstellar medium; and (iv) the propagation modeling; the paper is organized into three main parts. The positron injection spectrum and its uncertainties are derived in Sect. 2. We use up-to-date nuclear cross-sections and show the differences with older parameterizations. The Green functions associated with the positron propagation throughout the Milky Way are discussed in Sect. 3. Our slab model is mostly characterized by energy losses and diffusion caused by magnetic turbulences. The secondary positron flux at the Earth is presented in Sect. 4 with a range of variation that includes the effects discussed throughout the paper. We also confirm that considering the cosmic ray proton and $\alpha$ retro-propagation as well as diffusive reacceleration and convection has little effect on our results above a few GeV. Our results for the positron flux and its uncertainties agree with all the available measurements by different experimental collaborations (we recall that PAMELA does not provide, at the moment, the positron flux, but only the positron fraction). The positron flux, by itself, does not exhibit unusual features and is in basic agreement with the results of Moskalenko \& Strong (1998) and Porter et al. (2008). This could imply that both the HEAT excess and the PAMELA rise observed in the positron fraction, originate from dark matter annihilation, but we argue in Sect. 5 that electrons might also play an important role. Depending on the data set used to constrain the electron spectrum, the estimate of the positron fraction can exhibit quite different behaviors, which can affect the interpretation of the positron fraction data. We show that our predictions can indeed be consistent with the existing data of the positron fraction for a soft electron spectrum, compatible with fits to experimental data. Hard electron spectra, instead, definitely point toward the presence of an excess. We finally insist in Sect. 6, as a main result of our analysis, that the current uncertainties in the transport parameters translate into one order of magnitude uncertainties in the secondary positron spectrum.

\section{Production of positrons by spallation}

Secondary positrons are created by spallation of cosmic ray nuclei (mainly protons and helium nuclei) on interstellar matter (mainly hydrogen and helium). We compute $q_{\mathrm{e}^{+}}\left(\boldsymbol{x}, E_{\mathrm{e}}\right)$, the number of positrons of energy $E_{\mathrm{e}}$ created per unit volume at position $\boldsymbol{x}$, per unit time and per $\mathrm{GeV}$. The positron source term reads:

$$
\begin{aligned}
q_{\mathrm{e}^{+}}\left(\boldsymbol{x}, E_{\mathrm{e}}\right) & =4 \pi \sum_{\operatorname{targ}=\mathrm{H}, \mathrm{He}} \sum_{\text {proj= }=p, \alpha} n_{\mathrm{targ}}(\boldsymbol{x}) \\
& \times \int \Phi_{\text {proj }}\left(\boldsymbol{x}, E_{\text {proj }}\right) \times \mathrm{d} E_{\text {proj }} \times \frac{\mathrm{d} \sigma}{\mathrm{d} E_{\mathrm{e}}}\left(E_{\text {proj }} \rightarrow E_{\mathrm{e}}\right),
\end{aligned}
$$

where $\Phi_{\text {proj }}\left(x, E_{\text {proj }}\right)$ denotes the cosmic ray nucleon flux at position $\boldsymbol{x}, n_{\operatorname{targ}}(\boldsymbol{x})$ the number density of target nuclei, and $\mathrm{d} \sigma / \mathrm{d} E_{\mathrm{e}}$ the cross-section for the reactions creating positrons. We discuss these quantities this section.

\subsection{Spallation cross-sections for $\mathrm{p}+\mathrm{p} \rightarrow \mathrm{e}^{+}$}

We first focus on the differential cross-section for the production of positrons. This production occurs by means of a nuclear reaction between two colliding nuclei, yielding mainly charged pions $\pi^{ \pm}$and other mesons, for which positrons are one of the final products of the decay chain. There are four main possible collisions: cosmic ray (CR) proton on interstellar (IS) hydrogen or helium; CR alpha particle on IS proton or helium. For the sake of clarity, we present only the formulae for the proton-proton collisions, but include all four processes in our results on the positron spectra.

At energies below about $3 \mathrm{GeV}$, the main channel for production of positrons involves the excitation of a Delta resonance, which then decays into pions:

$\mathrm{p}+\mathrm{H} \rightarrow \mathrm{p}+\Delta^{+} \rightarrow\left\{\begin{array}{l}\mathrm{p}+\pi^{0} \\ \mathrm{n}+\pi^{+}\end{array}\right.$

The charged pions decay into muons, which subsequently decay into positrons. At higher energies direct production of charged pions proceeds with the process:

$\mathrm{p}+\mathrm{H} \rightarrow \mathrm{p}+\mathrm{n}+\pi^{+}$

Kaons may also be produced:

$\mathrm{p}+\mathrm{H} \rightarrow X+K^{ \pm}$

and the decay of kaons produces muons (63.44\%) and pions (20.92\%), which then decay into positrons as final products of their decay chain.

To compute the differential cross-section for the pionproduction processes, we need the probability $\mathrm{d} \sigma\left(E_{\mathrm{p}} \rightarrow\right.$ $\left.E_{\pi}\right) / \mathrm{d} E_{\pi}$ of a spallation of a proton of energy $E_{\mathrm{p}}$ yielding a pion with energy $E_{\pi}$ and the probability $\mathcal{P}\left(E_{\pi} \rightarrow E_{\mathrm{e}}\right)$ of such a pion eventually decaying into a positron of energy $E_{\mathrm{e}}$. This second quantity can be computed thanks to basic quantum electrodynamics. whereas several parameterizations of the first quantity can be found in Badhwar \& Stephens (1977), Tan \& Ng (1983) and Kamae et al. (2006). The production cross-section of positrons is then given by:

$$
\begin{aligned}
& \frac{\mathrm{d} \sigma}{\mathrm{d} E_{e}}\left(E_{\mathrm{p}} \rightarrow \pi^{+} \rightarrow E_{\mathrm{e}}\right)= \\
& \int \frac{\mathrm{d} \sigma}{\mathrm{d} E_{\pi}}\left(E_{\mathrm{p}} \rightarrow E_{\pi}\right) \times \mathrm{d} E_{\pi} \times \mathcal{P}\left(E_{\pi} \rightarrow E_{\mathrm{e}}\right) .
\end{aligned}
$$

Kamae et al. (2006) also provided a direct parameterization of the $\mathrm{p}+\mathrm{p} \rightarrow \mathrm{e}^{+}$reaction $^{1}$. All the afore mentioned parameterizations differ from each other and have been calibrated with different nuclear data sets. The default choice in our calculations, unless stated otherwise, is the Kamae et al. (2006) parameterization, which includes additional processes (especially resonances other than the Delta at low interaction energies) and has been calibrated with recent data. As stated in the original paper, we nevertheless warn that this parameterization relies on fits to Monte Carlo simulations and may be easilly affected by small uncertainties.

\footnotetext{
1 A few typos in the published version have been corrected with the kind help of the authors.
} 

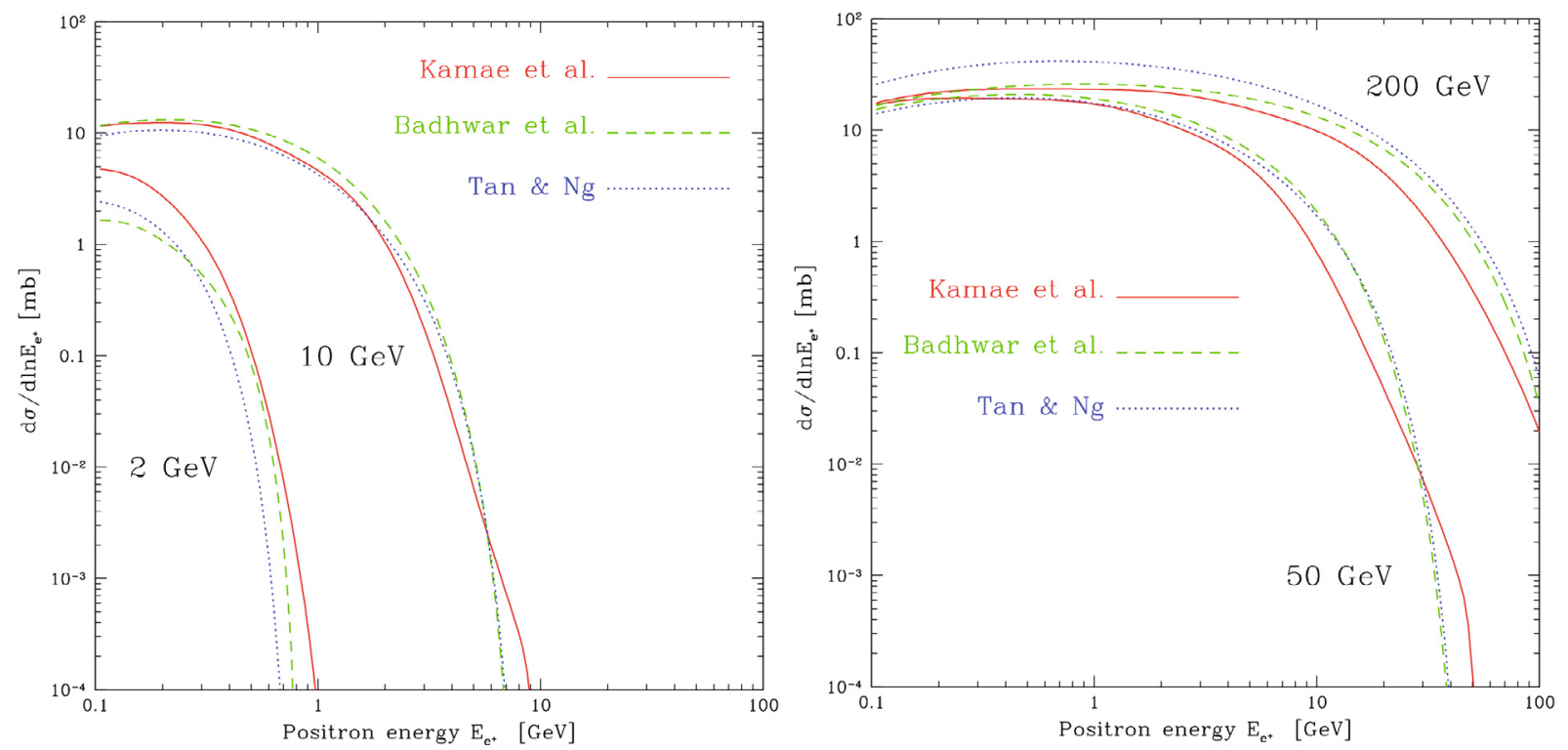

Fig. 1. Comparison between various parameterizations of the positron production cross-section at different incident proton energies.

The cross-section for the process involving kaons can be computed in a similar way to the calculation of direct pion production. The QED expressions for the production of positrons from the kaon are provided e.g. in Appendix D of Moskalenko \& Strong (1998).

In Fig. 1, we plot the cross-section for the positron production from the p-H scattering, as a function of positron energy. The incident proton energy is set to 2, 10 (left), 50 and 200 (right) $\mathrm{GeV}$. The three different plots at fixed proton energy correspond to the cross-section parameterizations of Kamae et al. (2006) (solid), Badhwar \& Stephens (1977) (dashed) and Tan $\&$ Ng (1983) (dotted). The differences between these plots vary with both incident proton and final positron energies. For protons of intermediate energies of 10 and $50 \mathrm{GeV}$, the flatter part of the cross-section (at low energies) varies only slightly between the different parameterizations. The Kamae et al. (2006) parameterization undershoots and then overshoots the other two models only in the high energy range, while for slow protons (see the $2 \mathrm{GeV}$ case), it provides more positrons. This is because both the multiple baryonic resonances around $1600 \mathrm{MeV}$ and the standard $\Delta(1232)$ state have been added into their model.

\subsection{Incident proton flux}

The proton flux $\Phi_{\mathrm{p}}\left(\boldsymbol{x}, E_{\mathrm{p}}\right)$ has been measured at the location of the Earth $\boldsymbol{x}=\boldsymbol{x}_{\odot}$ by several experiments. Various parameterizations of these measurements are found in the literature. In this analysis, we adopt the determinations of Shikaze et al. (2007) and Donato et al. (2001). The effect induced on the positron source term in Eq. (1) is displayed in Fig. 2, where we also show the effect arising from the different nuclear physics parameterizations discussed in the previous subsection. The solid lines refer to Kamae et al. (2006), the dashed lines to Badhwar \& Stephens (1977), and the dotted lines to Tan \& Ng (1983). For each set of curves, the thick lines are obtained for the proton spectrum parameterization of Donato et al. (2001), while the thin lines refer to Shikaze et al. (2007). Figure 2 illustrates that different parameterizations of the incident proton flux cause less significant uncertainty than the nuclear models. At low $(1 \mathrm{GeV})$ and high $\left(10^{3} \mathrm{GeV}\right)$ energies, the Shikaze et al. (2007)

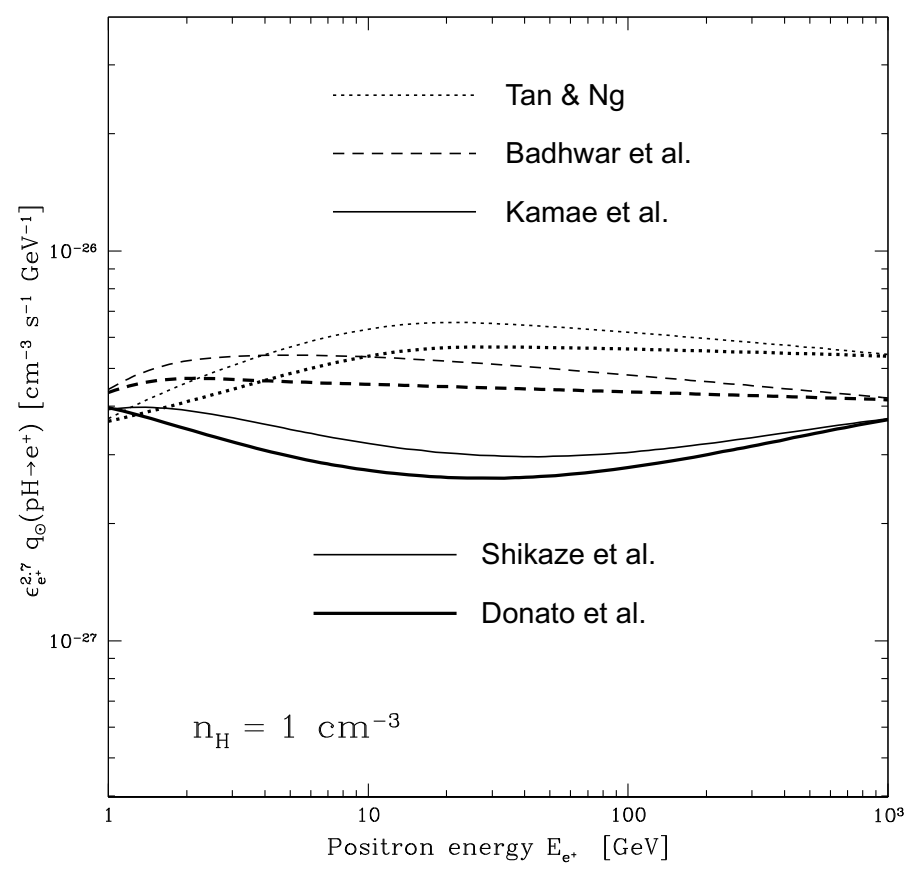

Fig. 2. Comparison of the effect due to different parameterizations for the cosmic ray proton spectra on the positron source term, as a function of the positrons energy. The additional effect induced by the different nuclear physics parameterizations is also shown. The galactic protons density is taken at 1 hydrogen atom per $\mathrm{cm}^{3}$.

and Donato et al. (2001) fluxes produce almost identical results on the proton flux, but at intermediate energies the differences are also negligible.

In contrast, the cross-section parameterizations produce more significant variations. Figure 2 shows that the model of Kamae et al. (2006) gives the faintest spectrum in all the energy range (from $1 \mathrm{GeV}$ to $1 \mathrm{TeV}$ ), while that of Tan \& $\mathrm{Ng}$ (1983) corresponds to the maximal spectrum. The most significant difference occurs at 20-30 GeV, of about a factor of two.

The spatial dependence of the proton flux is determined by solving the diffusion equation and normalizing the resulting flux 
to the solar value. This procedure will be referred to as retropropagation in the following. We find that retropropagation is not crucial and that one can safely approximate the proton flux as being homogeneous and equal to the solar value. This is because most positrons detected in the solar neighborhood have been created locally, over a region where the proton flux does not vary significally. This will be discussed further in the final section.

For the sake of simplicity, we show only the impact of the $\mathrm{pH}$ interaction on the determination of the positron spectrum, but we recall that our results in the subsequent sections refer to cosmic ray protons and $\alpha$ interacting with both IS hydrogen and helium with densities of $n_{\mathrm{H}}=0.9 \mathrm{~cm}^{-3}$ and $n_{\mathrm{He}}=0.1 \mathrm{~cm}^{-3}$ respectively. These average values for the hydrogen and helium densities are of course approximations based on local estimates, but we do not expect significant changes in the averaging within the kpc scale (Ferrière et al. 2007), which, as we show in the subsequent sections, is the relevant scale for the secondary positron propagation. Cross-sections for heavy nuclei were dealt with in the same way as in Norbury \& Townsend (2007).

\section{Propagation}

In the Galaxy, a charged particle travelling between its source and the solar neighborhood is affected by several processes. Scattering by magnetic fields leads to a random walk in both real space (diffusion) and momentum space (diffusive reacceleration). Particles may also be spatially convected away by the galactic wind (which induces adiabatic losses), and lose energy as they interact with either interstellar matter or the electromagnetic field and radiation of the Galaxy (by synchrotron radiation and inverse Compton processes). Above a few $\mathrm{GeV}$, the propagation of positrons in the Milky Way is dominated by space diffusion and energy losses.

In this paper, the diffusion coefficient is assumed to be homogeneous and isotropic, with a dependence on energy given by $K(E)=\beta K_{0}(\mathcal{R} / 1 \mathrm{GV})^{\delta}$, where the magnetic rigidity $\mathcal{R}$ is related to the momentum $p$ and electric charge $Z e$ by $\mathcal{R}=p c / Z e$. Cosmic rays are confined within a cylindrical diffusive halo of radius $R=20 \mathrm{kpc}$ and height $2 L$, their density vanishing at the boundaries $N(|z|=L, r)=N(z, r=R)=0$. As discussed below, the radial boundary has a negligible effect on the density of positrons in the Solar System. In this section, we do not consider the possibility of Galactic convection and diffusive reacceleration. These processes are taken into account in Sect. 4.2, where we demostrate that they have little effect. The convective wind is assumed to carry cosmic rays away from the Milky Way disk in the $z$ direction at a constant velocity $V_{\mathrm{c}}$. Diffusive reacceleration depends on the velocity $V_{\mathrm{a}}$ of the Alfvèn waves. The free model parameters are therefore the size $L$ of the diffusive halo, both the normalization $K_{0}$ and spectral index $\delta$ of the diffusion coefficient, the convective wind velocity $V_{c}$, and the Alfvèn velocity $V_{\text {a }}$ (see Sect. 3.3 for additional details). This model has been consistently used in several studies to constrain the propagation parameters (Maurin et al. 2001, 2002; Donato et al. 2002) and examine their consequences (Taillet \& Maurin 2003; Maurin $\&$ Taillet 2003) for the standard $\bar{p}$ flux (Donato et al. 2001), the exotic $\bar{p}$ and $\bar{d}$ fluxes (Maurin et al. 2004, 2006; Donato et al. 2004; Barrau et al. 2002, 2005; Bringmann \& Salati 2007), and also for positrons (Lavalle et al. 2008b; Delahaye et al. 2008). The reader is referred to Maurin et al. (2001) for a more detailed presentation and motivation of the framework.

\subsection{The Green function for positrons}

The propagation of positrons differs from that of the nuclei in several respects. Although space diffusion is an essential ingredient common to all cosmic ray species, positrons suffer mostly from inverse Compton and synchrotron energy losses, e.g. Moskalenko \& Strong (1998), whereas (anti-)protons are mostly sensitive to the galactic wind and the nuclear interactions as they cross the Milky Way disk. As a result, a positron line at source leads to an extended spectrum after its propagation. This disagrees with studies of most nuclear species for which, as first approximation, energy losses can be neglected. Consequently, the diffusion equation that leads to the positron number density $N$ per unit of volume and energy, with the source term $q_{\mathrm{e}^{+}}(\boldsymbol{x}, E)$, becomes:

$-K_{0}\left(\frac{E}{E_{0}}\right)^{\delta} \Delta N+\frac{\partial}{\partial E}\left\{\frac{\mathrm{d} E}{\mathrm{~d} t} N\right\}=q_{\mathrm{e}^{+}}(\boldsymbol{x}, E)$.

The first term is simply the diffusion coefficient written as $K(\mathcal{R}) \approx K_{0}\left(E / E_{0}\right)^{\delta}$, where $E_{0} \equiv 1 \mathrm{GeV}$ is used to maintain the correct units throughout the paper.

The synchrotron and inverse Compton losses can be written as $\mathrm{d} E / \mathrm{d} t(E)=-E^{2} /\left(E_{0} \tau_{E}\right)$. Defining a pseudo-time:

$\hat{t} \equiv \tau_{E} \frac{\left(E / E_{0}\right)^{\delta-1}}{1-\delta}$

and applying the following rescaling:

$\hat{N} \equiv\left(E / E_{0}\right)^{2} N \quad$ and $\quad \hat{q}_{\mathrm{e}^{+}}(\boldsymbol{x}, E) \equiv\left(E / E_{0}\right)^{2-\delta} q_{\mathrm{e}^{+}}(\boldsymbol{x}, E)$,

the diffusion equation can be rewritten as:

$\frac{\partial \hat{N}}{\partial \hat{t}}-K_{0} \Delta \hat{N}=\hat{q}_{\mathrm{e}^{+}}(\boldsymbol{x}, \hat{t})$,

which is formally identical to the well-known time-dependent diffusion equation (Bulanov \& Dogel 1974; Baltz \& Edsjö 1999).

It proves convenient to separate diffusion along the radial and vertical directions. Considering a source located at $\left(x, y, z, \hat{t}_{E_{\mathrm{S}}}\right)$ and detected at $\left(R_{\odot}, 0,0, \hat{t}_{E_{\mathrm{O}}}\right)$, the corresponding flux depends only on the radial relative distance $r=\left|\boldsymbol{r}_{\mathrm{S}}-\boldsymbol{r}_{\mathrm{O}}\right|$, the distance of the source from the plane $z=z_{\mathrm{S}}$ and the relative pseudo-time $\hat{\tau}=\hat{t}_{E_{\mathrm{O}}}-\hat{t}_{E_{\mathrm{S}}}$. When the radial boundary is taken into account, one has to use an expansion over Bessel functions (Bulanov \& Dogel 1974; Delahaye et al. 2008). However, in most situations it is safe to ignore the radial boundary. The Green function $\hat{\mathcal{G}}_{\odot}(r, z, \hat{\tau})$ of Eq. (8) is then given by:

$\hat{\mathcal{G}}_{\odot}(\hat{\tau}, r, z)=\frac{\theta(\hat{\tau})}{4 \pi K_{0} \hat{\tau}} \exp \left(-\frac{r^{2}}{4 K_{0} \hat{\tau}}\right) \times \mathcal{G}^{1 D}(z, \hat{\tau})$.

The radial behavior of the positron Green function encourages us to define the characteristic diffusion length:

$\lambda_{\mathrm{D}} \equiv \sqrt{4 K_{0} \hat{\tau}}$

This length defines the scale of the positron sphere, i.e, the region where most of the positrons detected at the Earth are produced. It depends on the injected $E_{\mathrm{S}}$ and detected $E_{\mathrm{O}}$ positron energies in terms of the pseudo-time difference $\hat{\tau}$. For GeV energies, $\lambda_{\mathrm{D}} \lesssim 5 \mathrm{kpc}$, typically, which defines the local character of the positron origin. The effect of boundaries along $z= \pm L$ appears in only $\mathcal{G}^{1 D}(z, \hat{\tau})$. For convergence properties, two distinct regimes are worth considering (Lavalle et al. 2007) 
1. when the extension $\lambda_{\mathrm{D}}$ of the positron sphere is smaller than the half-thickness $L$ of the diffusive halo, it is most appropriate to use the so-called electrical image formula (e.g. Baltz \& Edsjö 1999):

$\mathcal{G}^{1 \mathrm{D}}(z, \hat{\tau})=\sum_{n=-\infty}^{+\infty}(-1)^{n} \frac{\theta(\hat{\tau})}{\sqrt{4 \pi K_{0} \hat{\tau}}} \exp \left\{-\frac{\left(z_{n}-z\right)^{2}}{4 K_{0} \hat{\tau}}\right\}$,

where $z_{n}=2 L n+(-1)^{n} z$;

2. in the opposite situation, a more suitable expression is based on an analogy with the solution to the Schrödinger equation in an infinitely deep square potential: expansion of the solution over the eigenfunctions of the Laplacian operator:

$\mathcal{G}^{1 \mathrm{D}}(z, \hat{\tau})=\frac{1}{L} \sum_{n=1}^{+\infty} \mathrm{e}^{-K_{0} k_{n}^{2} \hat{\tau}} \phi_{n}(0) \phi_{n}(z)+\mathrm{e}^{-K_{0} k_{n}^{\prime 2} \hat{\tau}} \phi_{n}^{\prime}(0) \phi_{n}^{\prime}(z)$

where:

$$
\begin{aligned}
& \phi_{n}(z)=\sin \left[k_{n}(L-|z|)\right] ; \quad k_{n}=\left(n-\frac{1}{2}\right) \frac{\pi}{L} \text { (even) } \\
& \phi_{n}^{\prime}(z)=\sin \left[k_{n}^{\prime}(L-z)\right] \quad \text { and } \quad k_{n}^{\prime}=n \frac{\pi}{L} \text { (odd). }
\end{aligned}
$$

The true positron propagator for a monochromatic point source is related to Eq. (9) by

$\mathcal{G}_{\odot}^{\mathrm{e}^{+}}\left(E \leftarrow E_{\mathrm{S}}, r, z\right)=\frac{\tau_{E} E_{0}}{E^{2}} \times \hat{\mathcal{G}}_{\odot}\left(\hat{\tau}=\hat{t}_{E}-\hat{t}_{E_{\mathrm{S}}}, r, z\right)$.

The secondary positron flux at the Earth is then given by (considering the Earth as the origin of the coordinate system):

$$
\begin{aligned}
\phi_{\mathrm{e}^{+}}^{\odot}(E) & =\frac{\beta c}{4 \pi} \int_{E}^{\infty} \mathrm{d} E_{\mathrm{S}} \\
& \times \int_{\text {slab }} \mathrm{d}^{3} \boldsymbol{x}_{\mathrm{S}} \mathcal{G}_{\odot}^{\mathrm{e}^{+}}\left(E \leftarrow E_{\mathrm{S}}, r_{\mathrm{S}}, z_{\mathrm{S}}\right) q_{\mathrm{e}^{+}}\left(\boldsymbol{x}_{\mathrm{S}}, E_{\mathrm{S}}\right),
\end{aligned}
$$

where $\left|x_{\mathrm{S}}\right|^{2}=r_{\mathrm{S}}^{2}+z_{\mathrm{S}}^{2}$ is the squared distance from the source to the Earth, and $\beta$ is the positron velocity in units of the velocity of light.

\subsection{Analytical solution for a homogeneous source term}

The secondary positrons originate from spallation processes of cosmic rays off the interstellar gas, the latter being located mainly inside the galactic disk. If we assume that the cosmic ray fluxes and the gas are homogeneous inside the disk and place the radial boundaries at infinity (see Sect. 3.1), the spatial integral in Eq. (14) can be derived analytically, e.g. by implementing an infinite disk of thickness $\left[-z_{\max }, z_{\max }\right]$. The flux at the Earth simplifies into

$\phi_{\mathrm{e}^{+}}^{\odot}(E)=\frac{\beta c}{4 \pi} \int_{E}^{\infty} \mathrm{d} E_{\mathrm{S}} q_{\mathrm{e}^{+}}\left(E_{\mathrm{S}}\right) \times \frac{\tau_{E} E_{0}}{E^{2}} \times \eta\left(\lambda_{\mathrm{D}}\right)$,

where

$\eta\left(\lambda_{\mathrm{D}}\right)=\int_{-z_{\max }}^{+z_{\max }} \mathrm{d}^{3} \boldsymbol{x}_{S} \hat{\mathcal{G}}_{\odot}\left(\hat{\tau}=\hat{t}_{E}-\hat{t}_{E_{\mathrm{S}}}, r, z\right)$.

This quantity depends only on the characteristic scale $\lambda_{\mathrm{D}}$ and can be expressed as

$\eta\left(\lambda_{\mathrm{D}}\right)=\left(\begin{array}{l}\frac{1}{2} \sum_{n=-\infty}^{\infty}\left\{\operatorname{Erf}\left(\frac{z_{n}^{\max }}{\lambda_{\mathrm{D}}}\right)-\operatorname{Erf}\left(\frac{z_{n}^{\min }}{\lambda_{\mathrm{D}}}\right)\right\}, \\ \frac{2}{L} \sum_{n=1}^{\infty}(-1)^{n+1} \frac{\cos \left(k_{n}\left(L-z_{\max }\right)\right)}{k_{n}} \times \mathrm{e}^{-k_{n}^{2} \lambda_{\mathrm{D}}^{2} / 4} .\end{array}\right.$

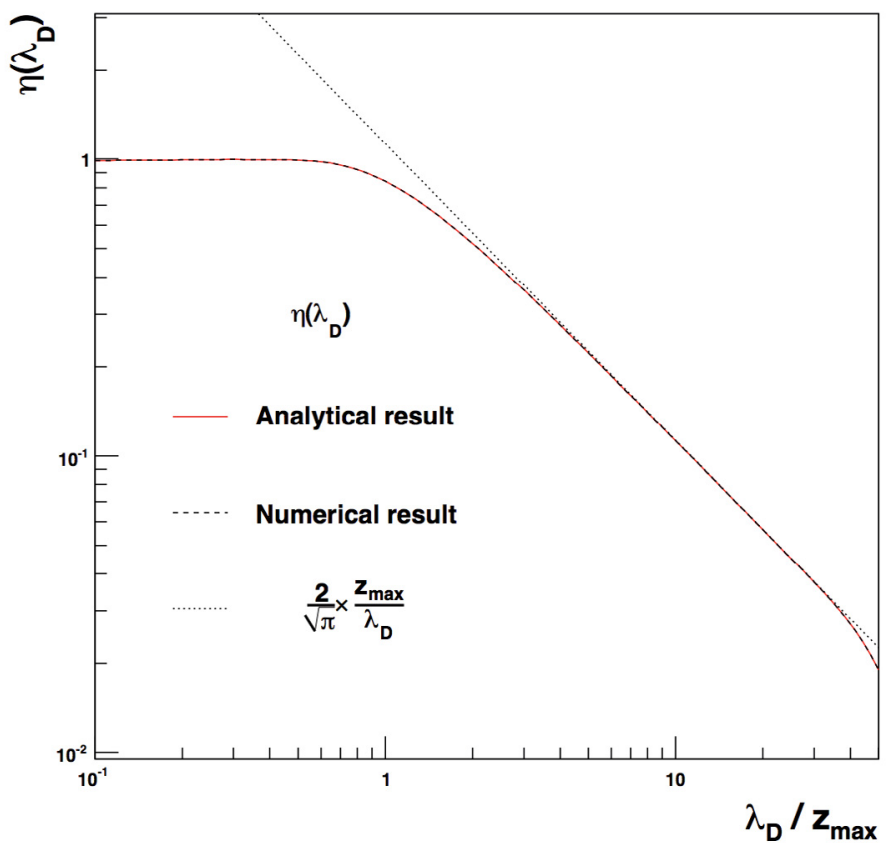

Fig. 3. The integral $\eta$ is plotted as a function of the ratio $\lambda_{\mathrm{D}} / z_{\max }$. Because $\eta$ can be interpreted as the the fraction of the positron sphere intersected by the Galactic disk, we infer that it should be unity for $\lambda_{\mathrm{D}} \ll z_{\max }$. In the converse regime, $\eta$ is proportional to the ratio $z_{\max } / \lambda_{\mathrm{D}}$. See text for further details.

The upper line corresponds to the electrical image solution, while the lower line is obtained by expanding the solution over the eigenfunctions of the Laplacian operator (see Sect. 3.1). Regarding the former case, we define $z_{n}^{\max } \equiv 2 n L+(-1)^{n} z_{\max }$ and $z_{n}^{\min } \equiv 2 n L-(-1)^{n} z_{\max }$ where $z_{\max }$ is defined to be $100 \mathrm{pc}$. The energy dependence of the solutions is hidden in the propagation length $\lambda_{\mathrm{D}}$. This simplification is helpful because it can provide an efficient way of checking the numerical spatial integral. Moreover, if the source term is shown to be almost homogeneous in the thin galactic disk, then this solution fully applies. Homogeneity is certainly justified in the energy range considered here but could break down at $\mathrm{TeV}$ energies.

In Fig. 3, $\eta$ is plotted as a function of the propagation length $\lambda_{\mathrm{D}}$. This parameter can be interpreted as the ratio of received to produced positrons. In the regime where $\lambda_{\mathrm{D}}$ is much smaller than the half-thickness $L$ of the diffusive halo, positron propagation occurs as if the diffusive halo were infinite. In this $3 \mathrm{D}$ limit, the propagator $\hat{\mathcal{G}}_{\odot}\left(\hat{\tau}=\hat{t}_{E}-\hat{t}_{E_{S}}, r, z\right)$ describes the probability of a positron detected at Earth originating in the location $r$ and $z$ and as such is normalized to unity. Secondary positrons are produced solely in the disk and $\eta$ equals by definition the fraction of the positron sphere filled by the disk. We therefore expect this fraction to be close to 1 when $\lambda_{\mathrm{D}}$ is smaller than $z_{\max }$ as the positron sphere becomes small enough to be embedded entirely inside the Galactic disk. In the converse situation, $\eta$ decreases approximately as

$\eta\left(\lambda_{\mathrm{D}} \gg z_{\max }\right) \simeq \frac{2}{\sqrt{\pi}} \frac{z_{\max }}{\lambda_{\mathrm{D}}}$.

Both behaviors are illustrated in Fig. 3.

\subsection{Propagation parameters}

The propagation parameters $\delta, K_{0}, L, V_{\mathrm{c}}$, and $V_{\mathrm{a}}$ are not measured directly. However, it is possible to determine the parameter 
Table 1. Typical combinations of diffusion parameters that are compatible with the B/C analysis (Maurin et al. 2001).

\begin{tabular}{cccccc}
\hline \hline Model & $\delta$ & $\begin{array}{c}K_{0} \\
{\left[\mathrm{kpc}^{2} / \mathrm{Myr}\right]}\end{array}$ & $\begin{array}{c}L \\
{[\mathrm{kpc}]}\end{array}$ & $\begin{array}{c}V_{\mathrm{c}} \\
{\left[\mathrm{km} \mathrm{s}^{-1}\right]}\end{array}$ & $\begin{array}{c}V_{\mathrm{a}} \\
{\left[\mathrm{km} \mathrm{s}^{-1}\right]}\end{array}$ \\
\hline MIN & 0.85 & 0.0016 & 1 & 13.5 & 22.4 \\
MED & 0.70 & 0.0112 & 4 & 12 & 52.9 \\
MAX & 0.46 & 0.0765 & 15 & 5 & 117.6 \\
\hline
\end{tabular}

As shown in Donato et al. (2004), these propagation models correspond respectively to minimal, medium, and maximal primary antiproton fluxes.

sets that are consistent with the observed properties of nuclei cosmic ray spectra, by comparing the observed spectra with the predictions of the diffusion model. In Maurin et al. (2001, 2002), the secondary-to-primary ratio $\mathrm{B} / \mathrm{C}$ is used to place constraints on the parameter space, and in Donato et al. (2002) the information provided by the cosmic ray radioactive species is studied. For the discussion below, it is convenient to isolate three sets of parameters labeled MIN, MED, and MAX, defined in Table 1. These configurations are named according to the primary antiproton signal yielded by dark matter species annihilating in the Milky Way halo (Barrau et al. 2002; Donato et al. 2004) and filling completely the diffusive halo. The half-thickness $L$ increases from 1 to $15 \mathrm{kpc}$ between models MIN and MAX. Notice that in the B/C analysis of Maurin et al. (2001), $L$ is shown to be correlated with the normalization $K_{0}$. Thick diffusive halos are associated with high diffusion coefficients and hence to high values of $\lambda_{\mathrm{D}}$. Inspired by Fig. 3, we anticipate that the secondary positron flux should have its lowest value in the MAX configuration and its highest for the MIN model.

\subsection{Energy losses}

A detailed analysis including all energy-loss processes is presented in Sect. 4.2. Here, we focus on the main processes for our energy range of interest. At energies higher than about $10 \mathrm{GeV}$, the most relevant energy losses are those caused by synchrotron radiation and inverse Compton (IC) scattering of the cosmic microwave background (CMB) and stars photons:

$$
-b^{\text {loss }}(\epsilon)=\frac{\epsilon^{2}}{\tau_{E}}=\frac{\epsilon^{2}}{\tau_{\mathrm{sync}}}+\frac{\epsilon^{2}}{\tau_{*}}+\frac{\epsilon^{2}}{\tau_{\mathrm{CMB}}} .
$$

Each energy-loss timescale $\tau$ can be calculated by means of both the Compton cross-section and the corresponding radiation-field energy density, as detailed in detail by Longair (1994). These Compton processes can indeed be computed in the Thomson limit. In the energy domain of interest here for positrons, say 10-100 GeV, the condition $\gamma_{\mathrm{e}^{+}} E_{\mathrm{ph}}<m_{\mathrm{e}}$, where $\gamma_{\mathrm{e}^{+}}$is the positron Lorentz factor and $E_{\mathrm{ph}}$ is the photon energy, is fulfilled for all relevant frequencies of the considered radiation fields.

By studying Eqs. (7) and (10), we note that $\tau_{E}$ has a significant effect on the positron propagation length: the higher the value of $\tau_{E}$, the farther is the origin of detected positrons from Earth. Since the Green function has a Gaussian dependence on the propagation scale, the importance of estimating accurately the $\tau_{E}$ parameter is reinforced, even though we expect the effect to be lower for secondaries than for primaries, for which the source term may, in some cases, exhibit a far more pronounced spatial dependency. In the following, we briefly discuss the different contributions to the energy-loss timescale in the high energy range. The $\mathrm{CMB}$ contribution is the most straightforward to

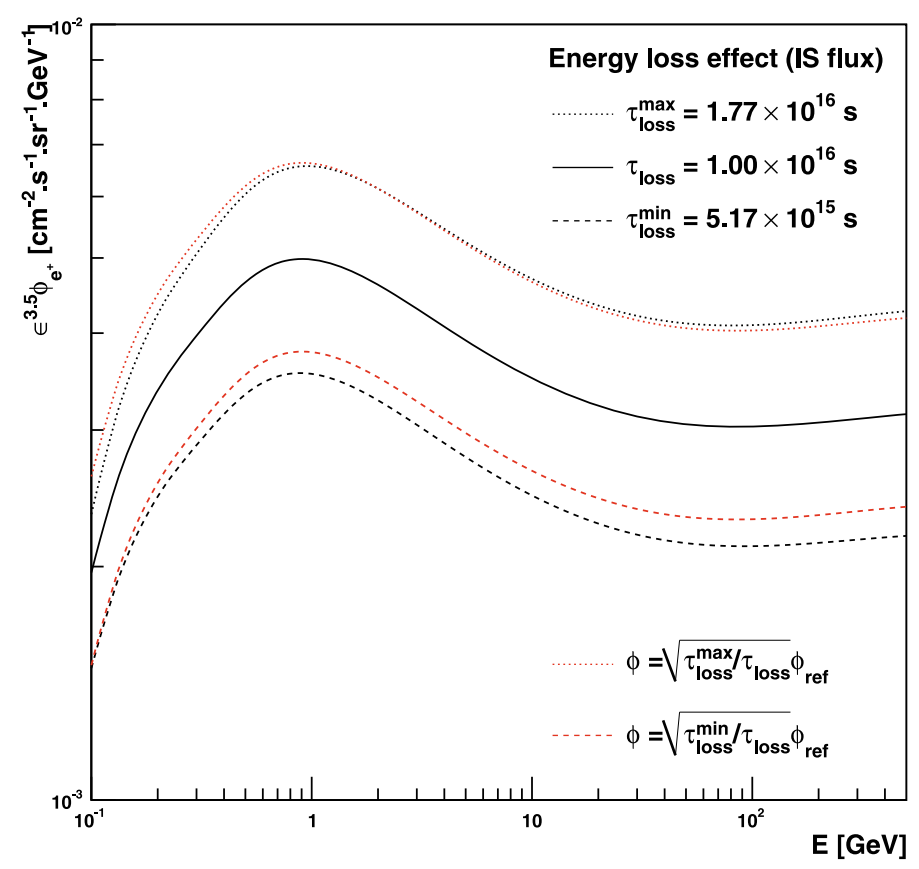

Fig. 4. Interstellar secondary positron flux $E^{3.5} \Phi_{\mathrm{e}^{+}}$as a function of the energy at Earth, for different values of the energy loss timescale $\tau_{E}$. The longer the timescale, the larger the flux. The scaling relation $\phi \propto \sqrt{\tau_{E}}$ is also reported.

compute. Adopting a mean CMB temperature of $T=2.725 \mathrm{~K}$, we readily determine $\tau_{\mathrm{CMB}}=3.77 \times 10^{16} \mathrm{~s}$.

For the synchrotron contribution, the local value of the magnetic field, which equals $\tau_{\text {sync }}$, remains unknown. As explained in Beck et al. (2003), there are two different methods of measurement. The first relies on the intensity of the synchrotron radiation from cosmic electrons and gives a value of $B \sim 4 \pm 1 \mu \mathrm{G}$ (Prouza \& Smida 2003). However, this value depends on the adopted model, particularly in terms of the cosmic ray electron spectrum estimation. The second method uses the Faraday rotation measurements of pulsar polarized emission, and yields $B \sim 1.8 \pm 0.3 \mu \mathrm{G}$ (Han et al. 2006). The two results are inconsistent but Beck et al. (2003) were able to identify further uncertainty in the second method, which, if the thermal electron density is anticorrelated with the magnetic field strength, produces a revised value of $B \in[1.5 ; 4] \mu \mathrm{G}$, and hence $\tau_{\text {sync }} \in$ $\left[2.47 \times 10^{16} ; 1.76 \times 10^{17}\right] \mathrm{s}$.

Finally, to evaluate the contribution of IC processes in the interstellar radiation field (ISRF), we rely on the study performed by Strong et al. (2000). They estimated the local value of the ISRF energy density, whose uncertainty can be inferred from its variation in value within the $2 \mathrm{kpc}$ around the solar position. This provides an average local ISRF energy density of $U_{\text {rad }} \sim 2 \pm 1 \mathrm{eV} \mathrm{cm}^{-3}$, hence $\tau_{\mathrm{IC}} \in\left[6.54 \times 10^{15} ; 1.96 \times 10^{16}\right] \mathrm{s}$.

The leading term caused by IC scattering by the ISRF (star and dust light) is clearly affected by the most significant uncertainty. By combining all the contributions, we derive an uncertainty range for the energy-loss timescale of $\tau_{E} \in[5.17 \times$ $\left.10^{15} ; 1.77 \times 10^{16}\right] \mathrm{s}$. As shown in Fig. 4, the uncertainty caused by $\tau_{E}$ does not exhibit a strong energy dependence. This can be understood from the expression of the positron Green function given in Eqs. (9) and (13), and from the fact that the source term of secondaries is close to be homogeneously distributed in the thin Galactic disk. From this, the secondary positron flux roughly scales as $\sqrt{\tau_{E}}$, as also illustrated in Fig. 4. Since $\tau_{E}$ determines the positron propagation scale, the related 


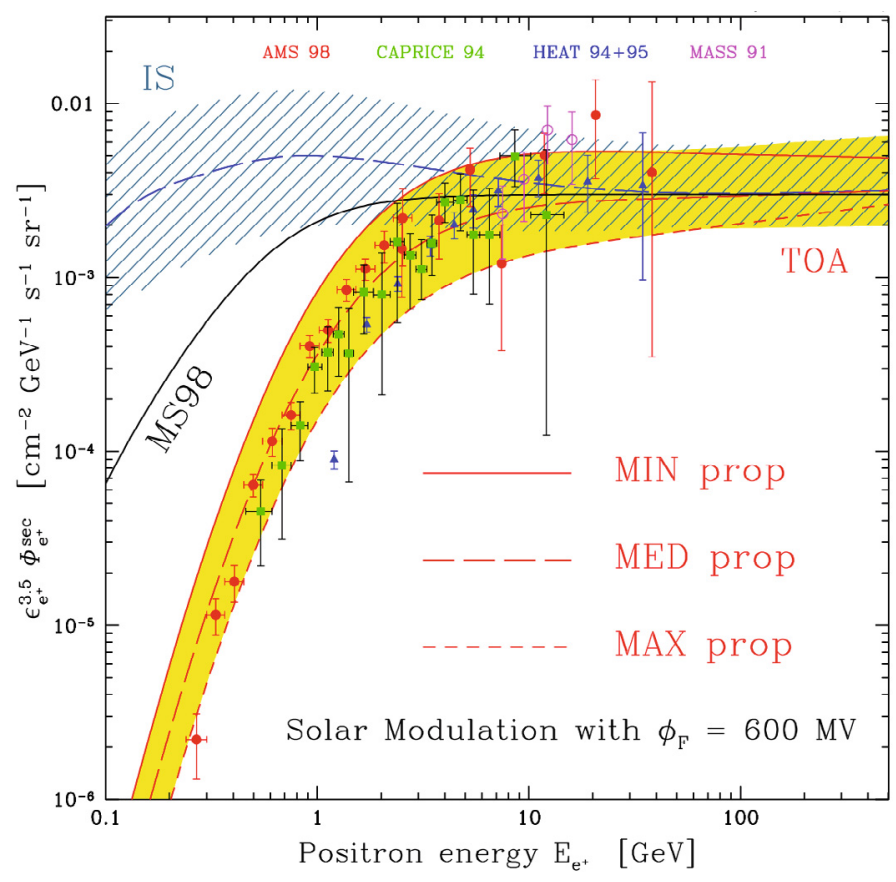

Fig. 5. Secondary positron flux as a function of the positron energy. The blue hatched band corresponds to the CR propagation uncertainty in the IS prediction, whereas the yellow strip refers to TOA fluxes. The longdashed curves feature our reference model with the Kamae et al. (2006) parameterization of nuclear cross-sections, the Shikaze et al. (2007) injection proton and helium spectra and the MED set of propagation parameters. The MIN, MED and MAX propagation parameters are displayed in Table 1. Data are taken from CAPRICE (Boezio et al. 2000), HEAT (Barwick et al. 1997), AMS (Aguilar et al. 2007; Alcaraz et al. 2000) and MASS (Grimani et al. 2002).

uncertainties should have a far stronger effect on the primary contributions with a far greater spatial (or time) dependency, such as for instance nearby astrophysical or exotic point sources. In the following, we have adopted as a standard the value of $\tau_{E}=1 \times 10^{16} \mathrm{~s}$.

We note that $\tau_{\text {sync }}$ and $\tau_{\star}$ are not expected to remain constant throughout the entire diffusive halo (Strong et al. 2000). However, as we discuss extensively in Sect. 4.1, most of the positrons detected at Earth with energies greater than a few $\mathrm{GeV}$, are typically of local origin. This is illustrated in Fig. 10, where $\tau_{E}=1 \times 10^{16} \mathrm{~s}$, for detected positrons of energy higher than $1 \mathrm{GeV}$ : more than $75 \%$ of the signal has originated within a distance of $2 \mathrm{kpc}$; the higher the detected energy, the higher this percentage. Since we are not interested in very low energy positrons, we can safely neglect spatial variations in the magnetic field $B$ and the ISRF energy density $U_{\text {rad }}$ discussed above.

\section{The positron flux and its uncertainties}

Figure 5 displays the calculated secondary positron flux modulated at solar minimum along with the most recent experimental data. We used a Fisk potential $\phi=600 \mathrm{MV}$ as applied in Perko (1987). The MIN, MED and MAX cases are illustrated by the red solid, long-dashed and short-dashed lines, respectively, while the yellow area denotes the uncertainty in the propagated flux caused by the uncertainty in the astrophysical parameters. The nuclear cross-section from Kamae et al. (2006) and the Shikaze et al. (2007) proton and helium fluxes were used. We adopted the MED prediction as our reference model. In the same figure, we also plot the interstellar flux. The upper long-dashed curve corresponds to the MED case whereas the slanted band indicates the uncertainty in the Galactic propagation parameters. The solid line shows the IS flux from Moskalenko \& Strong (1998). Below 100 GeV, the yellow uncertainty band is delineated by the MIN and MAX models. As discussed at the end of the previous section, the MIN (MAX) set of parameters yields the highest (lowest respectively) values for the secondary positron flux. Since we considered more than about 1600 different configurations compatible with the B/C ratio (Maurin et al. 2001), other propagation models become important in determining the extremes of the uncertainty band at higher energies. The maximal flux at energies above $100 \mathrm{GeV}$ does not correspond to any specific set of propagation parameters over the whole range of energies, as already noted in Delahaye et al. (2008), where the case of positrons produced by dark matter annihilation was studied.

From Fig. 5, we see that the variation in the propagation parameters induces an uncertainty in the positron flux, which reaches about one order of magnitude over the entire energy range considered here. It is a factor of 6 at $1 \mathrm{GeV}$, and smoothly decreases down to a factor of 4 or less for energies higher than $100 \mathrm{GeV}$. The agreement with experimental data is quite good at all energies within the uncertainty band. The Moskalenko \& Strong (1998) prediction of the IS secondary positron flux as parameterized by Baltz \& Edsjö (1999) is indicated by the black solid curve, and hardly differs from our reference model (long-dashed curve and MED propagation) above a few GeV. The HEAT data points are in good statistical agreement with this MED model.

The effects induced by different parameterizations of the nuclear production cross-sections and by the variation in the proton injection spectrum are shown in Fig. 6. In the left panel, we present the TOA positron fluxes calculated from the Tan \& $\mathrm{Ng}$ (1983) (dotted), Badhwar et al. (1977) (dashed), and Kamae et al. (2006) (solid) cross-section models for the MED propagation scheme and the Shikaze et al. (2007) proton and helium injection spectra. The Kamae et al. (2006) model leads systematically to the lowest flux. For positron energies $\lesssim 1 \mathrm{GeV}$, the three cross-section parameterizations differ by just a few percent, while the differences are significantly larger at higher energies. Figure 6 translates the uncertainties in the source term $q_{\mathrm{e}^{+}}$featured in Fig. 2. Consequently, the flux obtained at $10 \mathrm{GeV}$ with the Tan \& Ng (1983) or Badhwar et al. (1977) parameterization is a factor of 2 or 1.6, respectively, higher than the reference case (Kamae et al. 2006). This trend is confirmed at higher energies, although the differences between the various models are smaller above $200 \mathrm{GeV}$.

The uncertainties caused by the proton and helium spectrum parameterizations are the least relevant to this analysis. This is demonstrated in the right panel of Fig. 6, where we compare the positron flux of the reference model (solid lines) with the flux obtained when the Shikaze et al. (2007) parameterization of the incident spectra was replaced by that of Donato et al. (2001). The differences are at most $10-15 \%$ around $10 \mathrm{GeV}$, and are negligible in the lower and higher energy tails.

\subsection{Spatial origin of the positrons}

At every location in the Galaxy, the positron production by spallation is determined by the local flux of cosmic ray proton and helium projectiles. Their spatial distribution $\Phi(\boldsymbol{x}, E)$ was assumed to be constant and set equal to the value $\Phi_{\odot}(E)$ measured at the Solar System location. However, we note that these CR primaries also diffuse in the Milky Way, so that their flux 

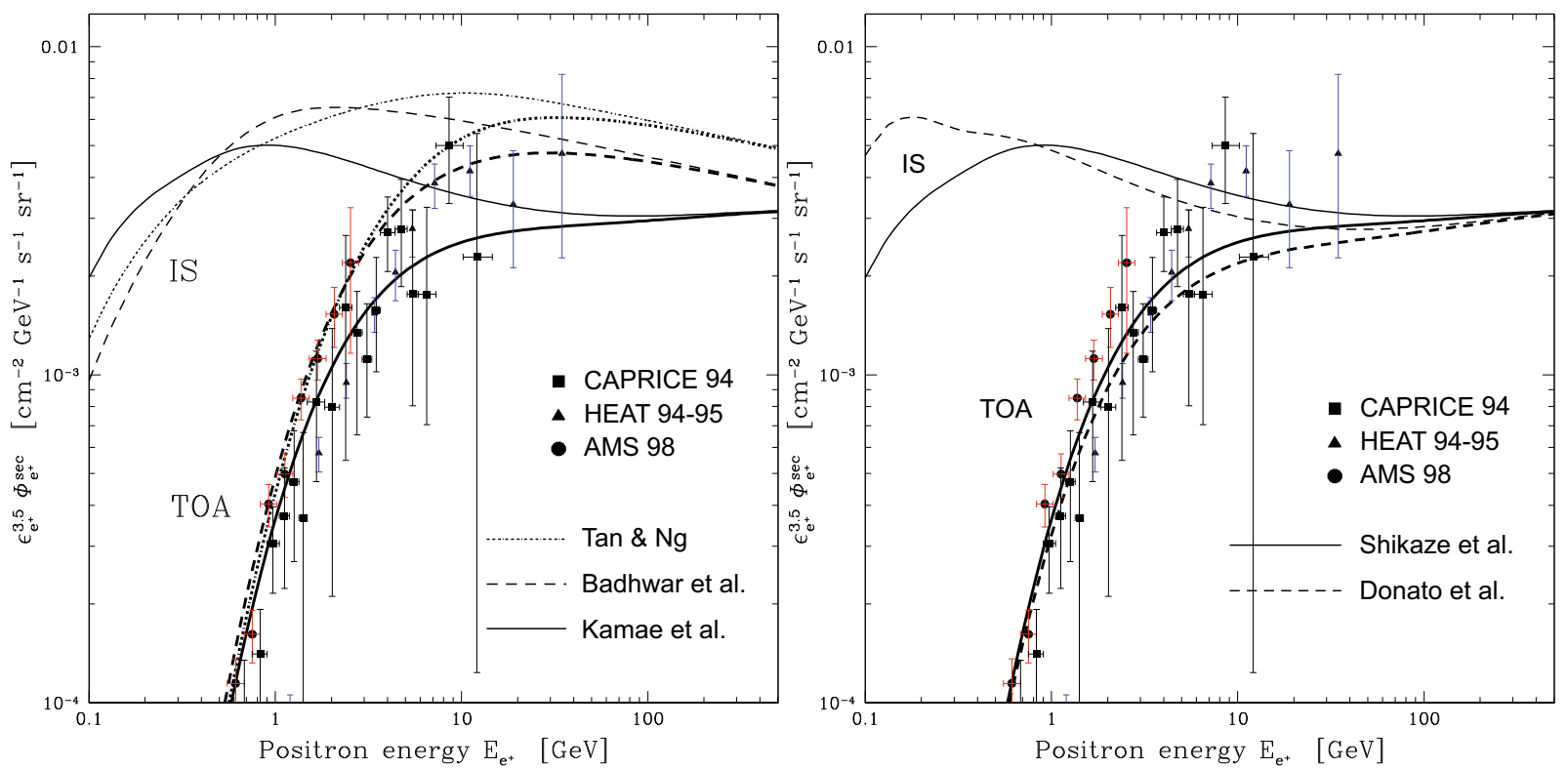

Fig. 6. Left: TOA and IS positron spectra for three different nuclear cross-section parameterizations: Kamae (solid), Badhwar (dashed) and Tan \& Ng (dotted). Right: TOA and IS positron spectra for two different proton fluxes: Shikaze (solid) and Donato (dashed). In all cases, diffusion parameters are set to the MED case of Table 1.

should exhibit a spatial dependence. The positron source term $q_{\mathrm{e}^{+}}$should vary accordingly inside the Galactic disk. The behavior of the proton and helium fluxes with radius $r$ can be inferred readily from their measured values $\Phi_{\odot}(E)$ once the propagation parameters are selected. This so-called retro-propagation was implemented in the original $\mathrm{B} / \mathrm{C}$ analysis by Maurin et al. (2001). The radial variation in the proton flux is presented in Fig. 7 for two quite different proton energies, and is found to be significant. This is why we questioned the hypothesis of a homogeneous positron production throughout the disk and found nevertheless that it remains viable in spite of the strong radial dependence of $\Phi(r, E)$.

This is because positrons reaching the Earth were mostly created locally, i.e., in a region in which the proton flux does not differ significantly from the local value $\Phi_{\odot}(E)$. We evaluated the contribution to the total signal from a disk of radius $r_{\text {source }}$ surrounding the Earth, which was modeled with the source term

$q_{\text {source }}(r, E)=q_{\mathrm{e}^{+}}(r, E) \times \Theta\left(r_{\text {source }}-r\right)$,

where $\Theta(x)$ is the Heaviside function and $r$ measures the radial distance from the Solar System. The positron flux yielded by $q_{\text {source }}$ is $\phi_{\mathrm{e}^{+}}^{\odot}\left(r_{\text {source }}, E\right)$, whose contribution to the total signal $\phi_{\mathrm{e}^{+}}^{\odot}(E)$ is plotted in Fig. 8 as a function of $r_{\text {source }}$, for several values of the positron energy $E$. Most of the positron signal originates at short distances, especially at high energy. At $1 \mathrm{TeV}$, more than $80 \%$ of the positrons are created within $1 \mathrm{kpc}$ while more than half of the $100 \mathrm{MeV}$ positrons come from less than $2 \mathrm{kpc}$. Energy losses are indeed quite efficient. They reduce the positron horizon strongly as the energy increases. This is why the CR proton and helium fluxes are close to their solar values when averaged over the positron horizon scale. Taking the retropropagation of projectile spectra into account has therefore little effect on the positron flux, as is clear in Fig. 9.

\subsection{Diffusive reacceleration and full energy losses}

Space diffusion and energy losses through inverse Compton scattering and synchrotron emission were the only processes that we

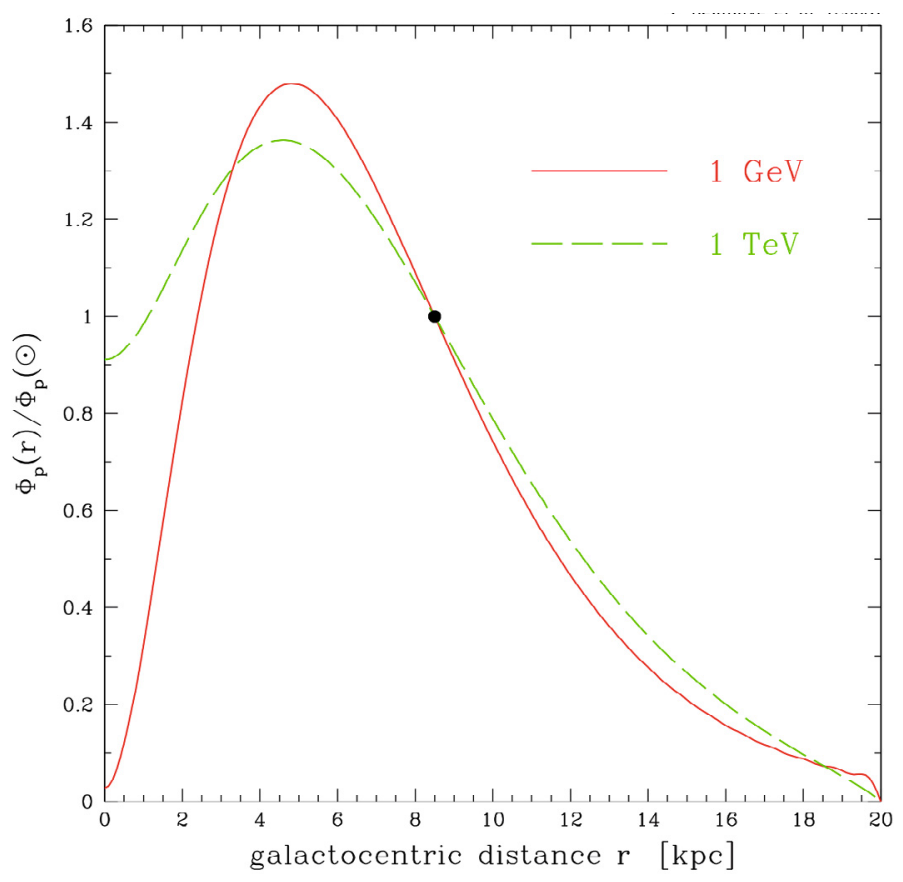

Fig. 7. Ratio of the proton flux at radius $r$ to the solar value from Shikaze et al. (2007). In this plot, retro-propagation has been taken into account, and all propagation effects of the MED configuration (i.e., convective wind, spallation, and diffusion) have been included. The dot refers to the Solar System position in the Galaxy.

had considered. We had neglected many other mechanisms that may also affect positron propagation. Galactic convection can sweep cosmic rays out of the diffusive halo and is associated with adiabatic energy losses. The drift of the magnetic turbulent field with respect to the Galactic frame with velocity $V_{\text {a }}$ induces both a diffusion in energy space and a reacceleration of particles. This so-called diffusive reacceleration was discussed in the original analysis of Moskalenko \& Strong (1998), but it does not appear in the fitting formula proposed by Baltz \& Edsjö (1999). 


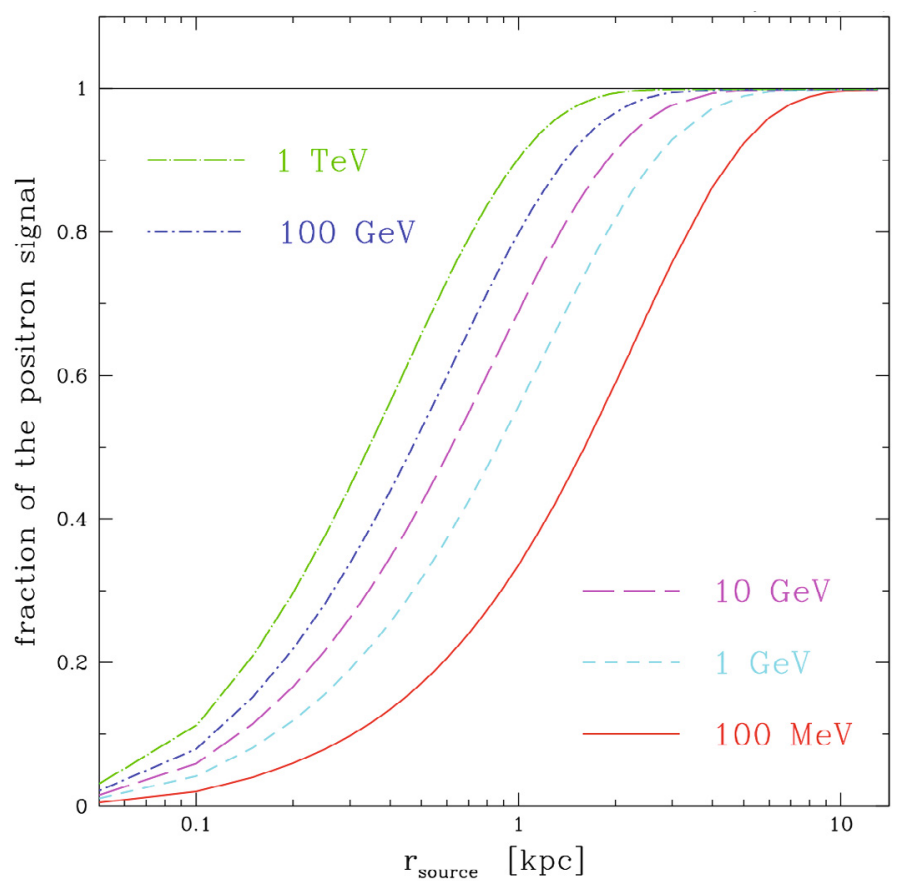

Fig. 8. Fraction of the positrons detected at the Earth which are produced within a disk of radius $r_{\text {source }}$. The larger the energy, the closer the source.

Finally, bremsstrahlung and ionization should come into play at low energies, below $\sim 1 \mathrm{GeV}$.

We note that once all these effects are considered, there is no longer an analytical solution to the diffusion equation, at least none that we know. Synchrotron and IC energy losses occur all over the diffusive halo, whereas the other energy-loss mechanisms as well as diffusive reacceleration are localized within the Galactic plane. A completely numerical approach is always possible of course - dealing for instance with a realistic distribution of gas, magnetic fields, and interstellar radiation fields - but this has never been our philosophy so far. Keeping calculations as analytical as possible has always been our guiding principle. We have therefore derived an approximate solution to the complete diffusion equation where the IC and synchrotron losses have been suitably renormalized and assumed to take place only in the disk. Obviously this is not fully correct, but a close inspection of the left panel of Fig. 10 and its solid curve indicates these values agree closely with the reference model featured in Fig. 5 by the long-dashed blue line. The diffusion equation may now be expressed as:

$$
\begin{aligned}
\nabla \cdot\{ & \left.-K_{0} \epsilon^{\delta} \nabla N+\boldsymbol{V}_{\mathrm{C}}(z) N\right\} \\
& +2 h \delta(z) \frac{\partial}{\partial \epsilon}\left\{b^{\text {loss }}(\epsilon) N-K_{\epsilon \epsilon} \frac{\partial N}{\partial \epsilon}\right\}=q_{\mathrm{e}^{+}}(\boldsymbol{x}, \epsilon)
\end{aligned}
$$

where $\epsilon=E / E_{0}$ and $E_{0}=1 \mathrm{GeV}$ as defined in Sect. 3.1. The convective wind varies with the vertical coordinate as:

$\boldsymbol{V}_{\mathrm{C}}(z)=\left\{\begin{aligned} V_{\mathrm{C}} \boldsymbol{u}_{z} & \text { if } z>0 \\ -V_{\mathrm{C}} \boldsymbol{u}_{z} & \text { if } z<0\end{aligned}\right.$

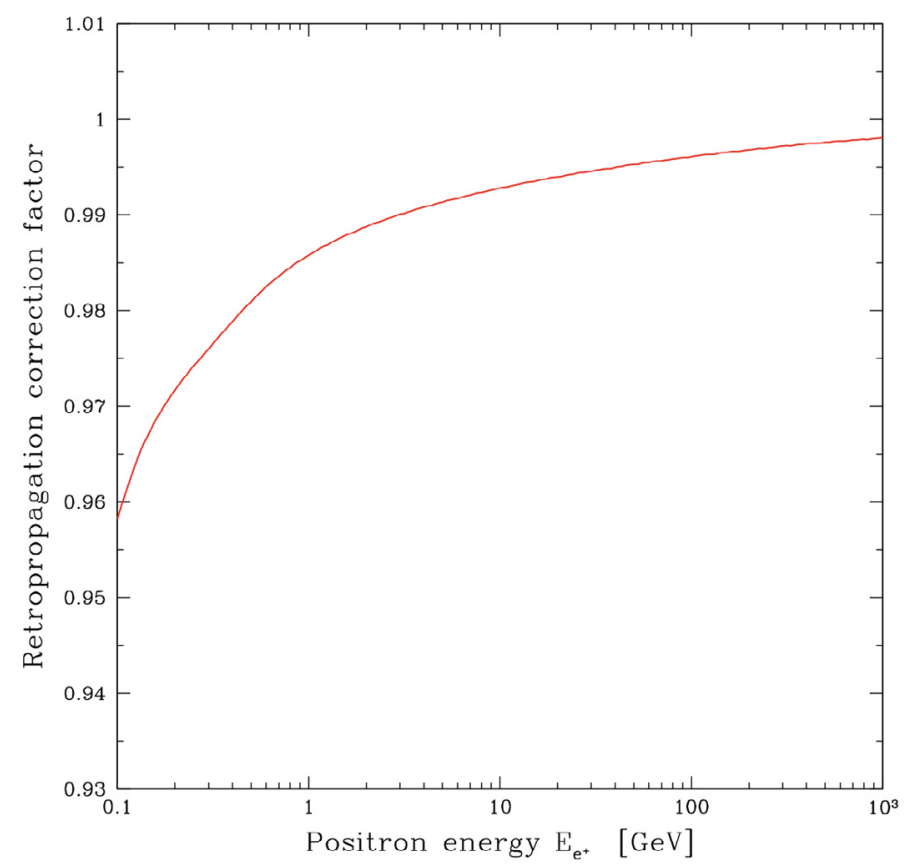

Fig. 9. Ratio of the positron flux computed with and without retropropagation, as a function of positron energy.

The energy-loss term is a combination of several contributions:

$$
-b^{\text {loss }}(\epsilon)=\left\{\begin{array}{lc}
\frac{\epsilon^{2}}{\tau_{E}} & \text { inverse Compton and synchrotron } \\
+\nabla \cdot V_{\mathrm{C}} \frac{p^{2}}{6 h \epsilon} & \text { adiabatic losses } \\
+K_{\mathrm{b}} n_{\mathrm{H}} \epsilon & \text { bremsstrahlung } \\
+K_{\mathrm{i}} n_{\mathrm{H}}\left\{3 \ln \left(\frac{E}{m_{e}}\right)+19.8\right\} & \text { ionisation. }
\end{array}\right.
$$

The values of the constants $K_{\mathrm{b}}$ and $K_{\mathrm{i}}$ can be found in Longair (1994). Diffusive reacceleration is taken care of by using a coefficient

$K_{\epsilon \epsilon}=\frac{2}{9} \frac{V_{\mathrm{a}}^{2}}{K_{0}} \epsilon^{2-\delta}$.

The positron density $N(r, z, \epsilon)$ is Bessel expanded. The coefficients $N_{i}(z, \epsilon)$ are determined all over the diffusive halo except for the normalizations $N_{i}(0, \epsilon)$. Each of these normalizations satisfies a diffusion equation in energy space, which we solve using a Crank-Nicholson semi-implicit method.

The solid line of Fig. 10 considers only space diffusion and energy losses by IC scattering and synchrotron emission. When these processes are supplemented by diffusive reacceleration, we derive the long-dashed curve with a noticeable bump at $\sim 3 \mathrm{GeV}$. Below that value, positrons are reaccelerated and their energy spectrum is shifted to higher energies. Above a few GeV, IC scattering and synchrotron emission dominate over diffusive reacceleration, inducing a shift in the spectrum towards lower energies. Positrons accumulate in an energy region where energy losses and diffusive reacceleration compensate each other, hence a visible bump which is already present in the analysis by Moskalenko \& Strong (1998). The short-dashed line is obtained by replacing diffusive reacceleration by Galactic convection. The wind is active at low energies, where space diffusion is slow. Positrons are drifted away from the Galaxy and their flux at the Earth is depleted. We note that diffusive reacceleration and Galactic convection were included separately by Lionetto et al. (2005) in 

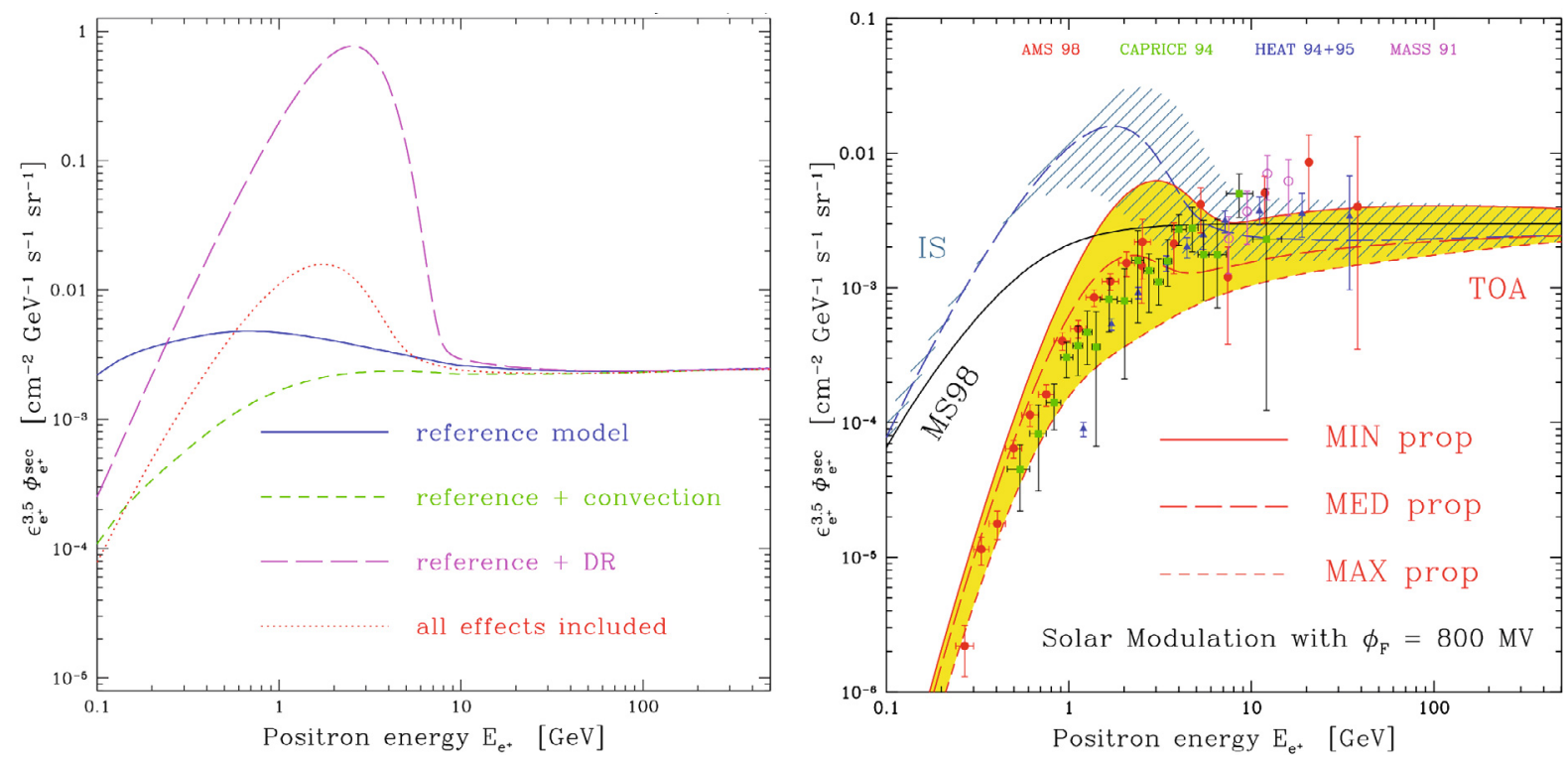

Fig. 10. Left panel: the reference model of Sect. 4 is featured here with various effects turned on or off. Space diffusion and energy losses from IC and synchrotron emission lead to the solid curve. When diffusive reacceleration is added, we get the long-dashed line and its spectacular bump around $3 \mathrm{GeV}$. The short-dashed curve is obtained by replacing diffusive reacceleration by galactic convection. The spectrum becomes depleted at low energies. Including all the processes yield the dotted line. Diffusive reacceleration and convection are both relevant below a few $\mathrm{GeV}$ and induce opposite effects. Right panel: the hatched blue (IS) and yellow (TOA) regions of Fig. 5 delineated by the MAX and MIN curves are featured here with all the effects included. Above a few GeV, we get the same results as before. Data are taken from CAPRICE (Boezio et al. 2000), HEAT (Barwick et al. 1997), AMS (Aguilar et al. 2007; Alcaraz et al. 2000) and MASS (Grimani et al. 2002).

their prediction of the positron spectrum, with the net result of either overshooting (diffusive reacceleration) or undershooting (galactic convection) the data. If we now incorporate both processes and add the various energy-loss mechanisms, we derive the dotted curve, which also contains a bump, although of far smaller amplitude. The bump cannot be distinguished from the solid line for energies above a few $\mathrm{GeV}$. This is the energy region where dark matter species are expected to distort the secondary spectrum and a calculation based solely on space diffusion and energy losses from IC scattering and synchrotron emission is perfectly safe.

Below a few $\mathrm{GeV}$, the situation becomes more complicated, several effects at stake modifying the blue hatched IS and yellow TOA uncertainty intervals in Fig. 5 as displayed in the right panel of Fig. 10. Reproducing the GeV bump of the IS flux with the data now requires a higher Fisk potential of $850 \mathrm{MV}$. The agreement seems reasonable below a few $\mathrm{GeV}$, although a more detailed investigation would require a refined solar modulation model.

\section{The positron fraction}

The question of whether a positron excess is being observed in cosmic ray measurements remains after many years, and is usually addressed in terms of the the so-called positron fraction, i.e., the quantity $\phi_{\mathrm{e}^{+}} /\left(\phi_{\mathrm{e}^{+}}+\phi_{\mathrm{e}^{-}}\right)$. This excess was pointed out by Moskalenko \& Strong (1998) when they derived their predictions of both the secondary positron flux and the primary plus secondary electron fluxes. This led to many possible interpretations, such e.g., a potential positron injection from dark matter annihilation in the Galaxy (e.g., Baltz \& Edsjö 1999) or from pulsars (e.g., Aharonian et al. 1995; Boulares 1989; Grimani 2007).

The PAMELA collaboration reported the positron fraction from 1.5 to $100 \mathrm{GeV}$ with unprecedented statistics quality
(Adriani et al. 2008). The PAMELA data are reported in Fig. 12, which we further explain in this section. Compared to the typical reference prediction by Moskalenko \& Strong (1998), a large excess appears. Our new predictions of the secondary positron flux and its theoretical uncertainties allow us to discuss in greater depth the interpretation of the excess positron fraction. We note first that we have shown in the previous sections that our predictions are consistent with the available positron measurements, excluding the PAMELA data since only their positron fraction data have so far become public. From these pre-PAMELA data, we therefore remark that an excess is hardly observed when considering the positron measurements only.

One of two crucial ingredients needed to derive the positron fraction is of course the electron flux. It was already noted by Moskalenko \& Strong (1998) that a change in the electron spectrum may affect the existence of an excess in the positron fraction. These authors compared the positron fraction obtained when using their own prediction of the electron spectrum, with that obtained when using the prediction of Protheroe (1982) based on the leaky box propagation model, and hence illustrated the difference due to the electrons. Today, we can attempt to take advantage of the higher quality existing electron data, and complement them with our theoretical calculation of the positron flux and its uncertainties.

It is nevertheless difficult to constrain the electron flux using the existing data, because they are available not only for limited energy ranges but also exhibit some differences in the absolute normalizations as well as the spectral shapes. Furthermore, the measurements available are mainly for energies below $\sim 50 \mathrm{GeV}$. For instance, the AMS electron data points of Aguilar et al. (2007) and Alcaraz et al. (2000), which are known to be among the most precise data sets to date, reach $\sim 30 \mathrm{GeV}$ only. Other complications are expected from astrophysical modeling arguments. For instance, it is likely that different spectral contributions are important at different energies, such as secondary 


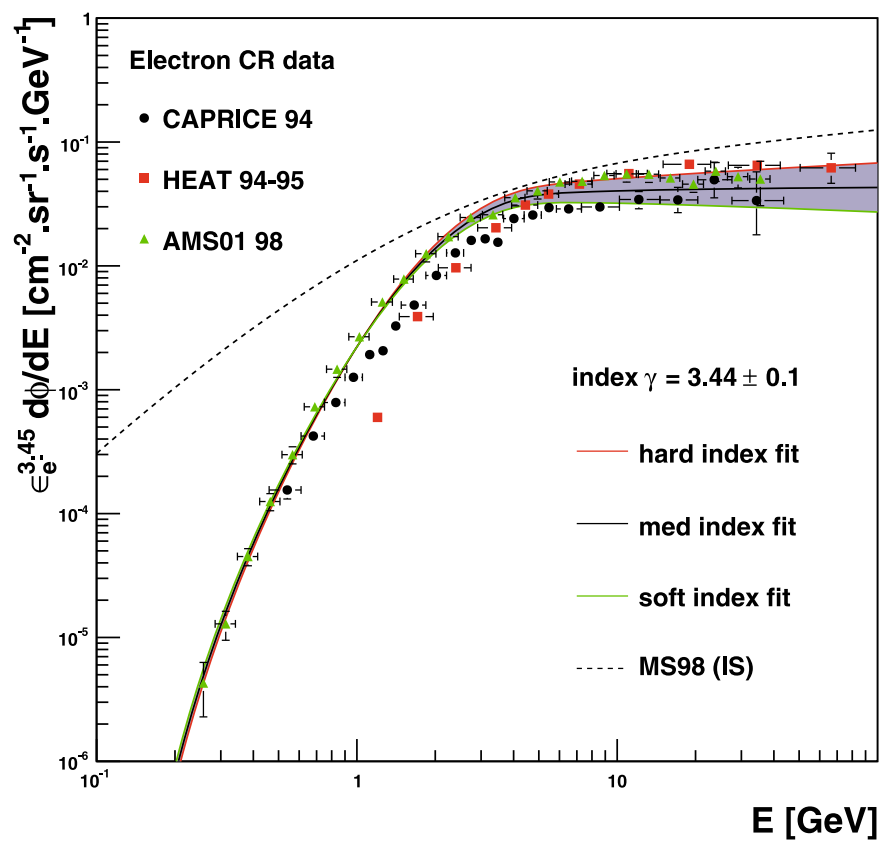

Fig. 11. Electron flux parameterization.

electrons at low energy, and primary electrons from local (distant) astrophysical cosmic ray sources at higher (intermediate, respectively) energies: this may imply that a blind fit to the data is not useful in the absence of any modeling insights. Although accounting for all these subtleties is beyond the scope of this paper, we can illustrate the importance of the electron spectrum by using a simple parameterization that is constrained reasonably up to $\sim 100 \mathrm{GeV}$, i.e., encompassing the PAMELA energy range, which fits the data at lower energies. We emphasize that predicting the electron flux is unnecessary to interpreting the positron contribution to the positron fraction as soon as electron data become available. Using the electron data directly instead of a model is also likely to provide a far more accurate description of any excess.

Making use of the electron data, we have therefore modeled an electron spectrum by a power law $\propto E^{-\gamma}$ at energies above a few $\mathrm{GeV}$ and up to $100 \mathrm{GeV}$, and allowed a scatter in the spectral index to account for the dispersion in the different sets of data. Because the permitted range of spectral indices that provide good fits is significant, we have applied restrictions by taking the central value and variance found by Casadei \& Bindi (2004), i.e., $\gamma=3.44 \pm 0.03$. Thus, an index range defined by $\gamma \pm 3 \sigma$ allows to encompass most of the available data below $100 \mathrm{GeV}$. The normalization of this electron spectrum as well as the low energy part have been adapted to the AMS data, which therefore includes the solar modulation effects. The choice of AMS is motivated by being likely to be the experimental setup least affected by systematic errors, but we emphasize that other setups are possible. Our parameterization of the electron flux is illustrated in Fig. 11, where the data from HEAT, CAPRICE, and AMS are presented. The dispersion observed in the data above a few $\mathrm{GeV}$ and below $\sim 100 \mathrm{GeV}$, i.e., over the entire PAMELA energy range, is reproduced well by taking a spectral index of $\gamma=3.44 \pm 0.1$, as mentioned above. To interpret the positron fraction measurements, we therefore considered two cases for the electron flux, a soft spectrum with index 3.54, and a hard spectrum with index 3.34. Finally, we note that we have also presented in Fig. 11 the interstellar electron model of Moskalenko \& Strong (1998), which, while those authors have since considerably improved their model, is still widely used as a reference for predicting the positron fraction: we note that this model clearly overshoots the AMS data above a few $\mathrm{GeV}$, and would therefore underestimate the positron fraction, should the electron data of AMS be close to the true flux and unaffected by systematics or unknown transient effects.

In Fig. 12, we show the positron fraction obtained for both the soft (left panel) and hard (right panel, respectively) electron spectra. For the positrons, we have used the Kamae nuclear cross-sections and the Shikaze proton and alpha injection spectra. The yellow band is bounded from below (above) by the MAX - short dashed curve - (MIN - solid curve -, respectively) set of propagation parameters, while the central longdashed curve represents the MED configuration. A solar modulation with $\phi=600 \mathrm{MV}$ has been applied to the positron flux, which corresponds to the level of solar activity during the data taking of AMS. In the same figure, we also report the positron fraction obtained with the positron flux of Moskalenko \& Strong (1998), but with our parameterization of the electron flux.

We see that, in the hard index case, a sizeable excess is present in the high energy tail. The MED reference curve is marginally compatible with the HEAT and AMS data above 10-20 GeV, which instead lies closer to the upper border of our predictions, thus favoring the MIN model, which is consistent with our predictions of the secondary positron flux (cf. Fig. 5), should the positron flux be dominated by a single secondary contribution. Therefore, when the theoretical uncertainties are considered, a clear assessment of an excess is not statistically significant on the basis of the HEAT and AMS data alone, apart from the $2 \sigma$ tension with the AMS data point at $12 \mathrm{GeV}$. Nevertheless, in the case of the PAMELA data, the MED reference flux is clearly incompatible with the experimental determinations for energies above $10 \mathrm{GeV}$. Even when theoretical uncertainties in the positron flux are taken into account, an excess is probably present for a hard electron spectrum.

When using the soft electron parameterization instead, we see that although an excess might still be apparent, its amplitude has strongly decreased, making it of least statistical relevance. The MED model indeed reproduces all the data-sets well from a few $\mathrm{GeV}$ up to $40 \mathrm{GeV}$ and a deviation is present for the last two bins of PAMELA, where the error bars are large due to reduced statistics. The PAMELA data may therefore be indicative of an excess also for a soft electron spectrum and energies above $50 \mathrm{GeV}$, but once the theoretical uncertainties on secondary positrons and statistical fluctuations in the data are taken into account, the amplitude of this excess is of least relevance. This implies that, for a soft electron spectrum, the secondary positron yield might still represent a very important contribution to the entire cosmic positron flux. We note, however, that below 4 $\mathrm{GeV}$, the MED configuration appears to disagree with the HEAT and AMS data, which would favor the MIN configuration.

Thus, we have attempted to discuss the positron fraction data by considering two different parameterizations for the electron flux, both consistent with the data below $100 \mathrm{GeV}$. If we had considered other fit parameters that were also consistent with the data, by modifying for instance the normalizations so as to ensure that they remained correlated with the spectral indices ${ }^{2}$, we

\footnotetext{
2 A correlation between the normalization and the spectral index obviously appears in the case of a single power law fit, but this would have absolutely no physical meaning if there actually were different spectral contributions - secondary, far primary, local primary - at different energies to the electron flux. A multi-component fit would break this correlation.
} 

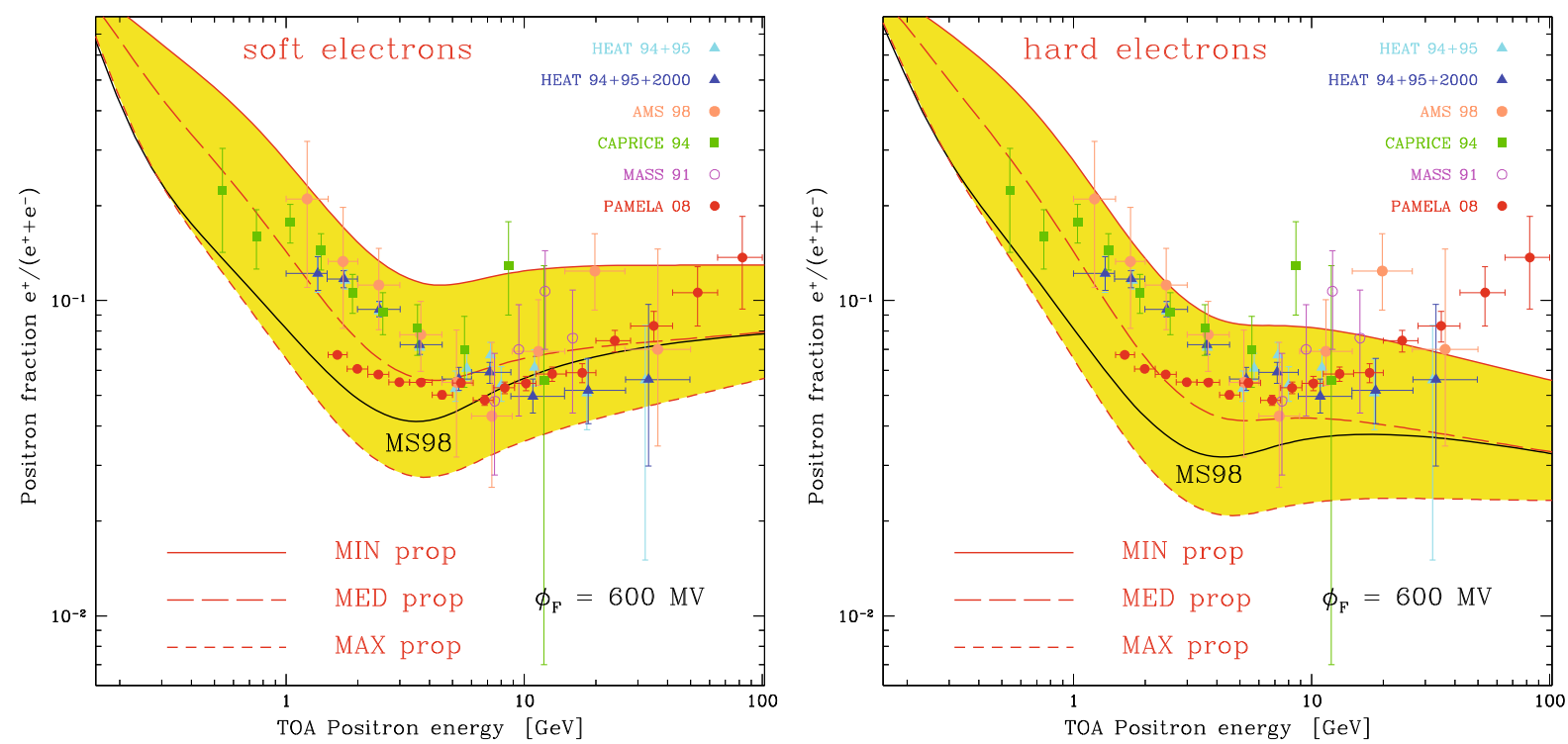

Fig. 12. Positron fraction as a function of the positron energy, for a soft (left panel) and hard (right panel) electron spectrum. Data are taken from CAPRICE (Boezio et al. 2000), HEAT (Barwick et al. 1997), AMS (Aguilar et al. 2007; Alcaraz et al. 2000), MASS (Grimani et al. 2002) and PAMELA (Adriani et al. 2008).

would have found quite different results opening up more or less the banana shape characterizing our predictions in Fig. 12. We therefore emphasize strongly that it is difficult to interpret the origin of the positrons observed in the positron fraction without comparing secondary positron predictions with the positron data, or a precise measurement of the electron flux. This requires access to both the electron and positron data separately, which have not yet been released in the case of PAMELA. With this in mind, it may be potentially unsafe to infer strong statements about the possible nature of any excess, since its amplitude and shape depend strongly on the underlying assumptions, which are not well constrained at the moment.

To conclude this section, we recall that in the previous sections we have shown that our theoretical predictions of the secondary positron flux perfectly agree with the available data (PAMELA data for the electron and positron components separately are not yet available), especially when the theoretical uncertainties are properly taken into consideration. We have shown in this section that the electrons play a very important role in the interpretation of the positron fraction released by the PAMELA collaboration. The scatter in the experimental measurements of the electron flux does not allow us, at the moment, to characterize fully the amplitude and shape of any positron excess with respect to the secondary yield. We have provided a critical illustration of this by using two different parameterizations of the electron flux (hard and soft cases) that are consistent with the data below $100 \mathrm{GeV}$, from which we have shown that the amplitude of an excess might be much smaller than expected from the positron/electron model of Moskalenko \& Strong (1998). Even if small, this excess could originate from additional astrophysical processes or dark matter annihilation. This implies that it is essential to estimate and understand the theoretical uncertainties affecting the background, as we have attempted in this paper.

\section{Conclusions}

Our aim has been to compute a correct estimation of the astrophysical positron flux and the corresponding uncertainties.
First, we have compared the various models available for the interstellar secondary positron production. It has been shown that more positrons are expected when the proton flux from Shikaze et al. (2007) is used, as compared to the case proposed by Donato et al. (2001). Moreover, for a given proton flux, the three positron production cross-sections we have considered produce different results: below a few $\mathrm{GeV}$, the parameterization of Badhwar et al. (1977) gives more positrons, whereas above a few $\mathrm{GeV}$, the model of Tan \& Ng (1983) predicts a higher positron production. At any energy, the parameterization of Kamae et al. (2006) produces the lowest amount of positrons.

Concerning the propagation of the positrons in the interstellar medium, we have used a Green function approach that led us to disregard convection and diffusive reacceleration: we have specifically included diffusion and energy losses due to inverse Compton scattering on cosmic microwave background photons and synchrotron radiation. Nevertheless, this analytical method allowed us to scan our $\sim 1600$ sets of propagation parameters compatible with the boron to carbon ratio measurements, and therefore to determine astrophysical uncertainties in the positron flux predictions. We showed that varying the diffusion parameters does not have the same effect as for primary positrons (Delahaye et al. 2008). For exotic positrons created in the Dark Matter halo, the thickness of the slab $2 L$ was the most relevant parameter because the increase of the diffusion zone implies the increase of the number of sources, whereas for secondary positrons - which are created in the Galactic disk only - the most relevant parameter is the diffusion constant $K_{0}$. Therefore, we expect the sets of parameters that basically maximize the primary positron flux to minimize the flux of secondary positrons, and vice versa.

We also showed that, because of energy losses during propagation, most of the positrons detected at the Earth have been created in the nearby $2 \mathrm{kpc}$ : this is the reason why we could safely neglect the variation in proton flux in the Galaxy. By solving the complete equation Eq. (22) with a numerical technique, we proved that all the other effects (convection, reacceleration, and other losses) can safely be neglected below $10 \mathrm{GeV}$ and that our method is valid. 
Finally, and this is our most important result, our estimation of the positron flux is compatible with all available data. This does not mean that there is no exotic positron contribution, since we have not tried to fit the data with a single diffusion model. However, this shows that one should be cautious before claiming that there is any excess in present data. Regarding a possible excess in the positron fraction, we have also clearly shown that the electron flux plays a role that is as important as that of positrons. This might sound tautological because the positron fraction is no more than a ratio, but so much energy is involved in support to this that we repeat this point. Figure 12 provides compelling visual argument.

The released PAMELA data (Adriani et al. 2008) show a clear increase in the positron fraction for energies above $10 \mathrm{GeV}$. From our analysis, whose objective is an accurate determination of the positron flux, we derive the conclusion that an excess is clear for a hard electron spectrum, while for a soft electron spectrum the rise in the positron fraction may be explained by the standard secondary production. By considering in turn the various parameters, we find in general that the PAMELA measurements are in excess of what a pure secondary component would yield. Nevertheless, if the electron spectrum is soft, most of the PAMELA data points are aligned with our MED prediction. We note also that, in that case, the two last energy bins feature an increase, but the experimental uncertainties are large there and a presence of an excess is, in this case, currently not statisticlly significant.

More insight into these issues will therefore require, from the theoretical side, a revised understanding also of the electron flux, including the determination of its uncertainties, and from the experimental side, the separate provision of the electron and positron fluxes, to allow more robust comparison of theoretical predictions with the data. In addition, the upcoming data on cosmic rays above $10 \mathrm{GeV}$ will allow us to reduce considerably the theoretical uncertainties in all cosmic ray fluxes and help us to elucidate the experimental status of the so-called excess in the positron spectrum.

Acknowledgements. Work supported by research grants funded jointly by Ministero dell'Istruzione, dell'Università e della Ricerca (MIUR), by Università di Torino (UniTO), by Istituto Nazionale di Fisica Nucleare (INFN) within the Astroparticle Physics Project, by the Italian Space Agency (ASI) under contract $\mathrm{N}^{\circ} \mathrm{I} / 088 / 06 / 0$ and by the French Programme National de Cosmologie. T.D. also acknowledges the International Doctorate on AstroParticle Physics (IDAPP) program. R.L. acknowledges financial support from the Comisión Nacional de Ciencia y Tecnología (CONICYT) from Chile (Grant $\mathrm{N}^{\circ}$ : BECAS-DOC-BIRF2005-00) and IDAPP.

\section{References}

Adriani, O., et al. 2008, Nature, submitted [arXiv:0810.4995]

Aguilar, M., Alcaraz, J., Allaby, J., et al. 2007, Phys. Lett. B, 646, 145
Aharonian, F. A., Atoyan, A. M., \& Voelk, H. J. 1995, A\&A, 294, L41 Alcaraz, J., Alpat, B., Ambrosi, G., et al. 2000, Phys. Lett. B, 484, 10 Badhwar, G. D. \& Stephens, S. A. 1977, in International Cosmic Ray Conference, International Cosmic Ray Conference, 1, 398

Badhwar, G. D., Golden, R. L., \& Stephens, S. A. 1977, Phys. Rev. D, 15, 820

Baltz, E. A., \& Edsjö, J. 1999, Phys. Rev. D, 59, 023511

Barrau, A., Boudoul, G., Donato, F., et al. 2002, A\&A, 388, 676

Barrau, A., Salati, P., Servant, G., et al. 2005, Phys. Rev. D, 72, 063507

Barwick, S. W., Beatty, J. J., Bhattacharyya, A., et al. 1997, ApJ, 482, L191

Beatty, J. J., Bhattacharyya, A., Bower, C., et al. 2004, Phys. Rev. Lett., 93, 241102

Beck, R., Shukurov, A., Sokoloff, D., \& Wielebinski, R. 2003, A\&A, 411, 99

Boezio, M., Carlson, P., Francke, T., et al. 2000, ApJ, 532, 653

Boulares, A. 1989, ApJ, 342, 807

Bringmann, T., \& Salati, P. 2007, Phys. Rev. D, 75, 083006

Bulanov, S. V., \& Dogel, V. A. 1974, Ap\&SS, 29, 305

Casadei, D., \& Bindi, V. 2004, ApJ, 612, 262

Delahaye, T., Lineros, R., Donato, F., Fornengo, N., \& Salati, P. 2008, Phys. Rev. D, 77, 063527

Donato, F., Fornengo, N., \& Salati, P. 2000, Phys. Rev. D, 62, 043003

Donato, F., Maurin, D., Salati, P., et al. 2001, ApJ, 563, 172

Donato, F., Maurin, D., \& Taillet, R. 2002, A\&A, 381, 539

Donato, F., Fornengo, N., Maurin, D., Salati, P., \& Taillet, R. 2004, Phys. Rev. D, 69, 063501

Donato, F., Fornengo, N., \& Maurin, D. 2008, Phys. Rev. D, 78, 043506

Ferrière, K., Gillard, W., \& Jean, P. 2007, A\&A, 467, 611

Grimani, C. 2007, A\&A, 474, 339

Grimani, C., Stephens, S. A., Cafagna, F. S., et al. 2002, A\&A, 392, 287

Han, J. L., Manchester, R. N., Lyne, A. G., Qiao, G. J., \& van Straten, W. 2006, ApJ, 642, 868

Kamae, T., Karlsson, N., Mizuno, T., Abe, T., \& Koi, T. 2006, ApJ, 647, 692

Lavalle, J., Pochon, J., Salati, P., \& Taillet, R. 2007, A\&A, 462, 827

Lavalle, J., Nezri, E., Ling, F., Athanassoula, L., \& Teyssier, R. 2008a, ArXiv e-prints

Lavalle, J., Yuan, Q., Maurin, D., \& Bi, X.-J. 2008b, A\&A, 479, 427

Lionetto, A. M., Morselli, A., \& Zdravkovic, V. 2005, J. Cosmol. Astro-Part. Phys., 9, 10

Longair, M. S. 1994, High Energy Astrophysics: Stars, the galaxy and the interstellar medium 2nd edn. (Cambridge: Cambridge University Press), 2

Maurin, D., \& Taillet, R. 2003, A\&A, 404, 949

Maurin, D., Donato, F., Taillet, R., \& Salati, P. 2001, ApJ, 555, 585

Maurin, D., Taillet, R., \& Donato, F. 2002, A\&A, 394, 1039

Maurin, D., Taillet, R., Donato, F., et al. 2004, Research Signposts,

Recent Research Developments in Astronomy and Astrophys., 2, 193 [arXiv: astro-ph/021211]

Maurin, D., Taillet, R., \& Combet, C. 2006, ArXiv Astrophysics e-prints

Moskalenko, I. V., \& Strong, A. W. 1998, ApJ, 493, 694

Norbury, J. W., \& Townsend, L. W. 2007, Nuclear Instrum. Methods Phys. Res. B, 254, 187

Perko, J. S. 1987, A\&A, 184, 119

Picozza, P., Galper, A. M., Castellini, G., et al. 2007, Astropart. Phys., 27, 296

Porter, T. A., Moskalenko, I. V., Strong, A. W., Orlando, E., \& Bouchet, L. 2008, ApJ, 682, 400

Protheroe, R. J. 1982, ApJ, 254, 391

Prouza, M., \& Smida, R. 2003, A\&A 410, 1

Shikaze, Y., Haino, S., Abe, K., et al. 2007, Astropart. Phys., 28, 154

Strong, A. W., Moskalenko, I. V., \& Reimer, O. 2000, ApJ, 537, 763

Taillet, R., \& Maurin, D. 2003, A\&A, 402, 971

Tan, L. C., \& Ng, L. K. 1983, J. Phys. G Nucl. Phys., 9, 1289 